Document downloaded from:

http://hdl.handle.net/10251/156655

This paper must be cited as:

Guijarro, L.; Pla, V.; Vidal Catalá, JR.; Naldi, M. (2019). Competition in data-based service provision: Nash equilibrium characterization. Future Generation Computer Systems. 96:3550. https://doi.org/10.1016/j.future.2019.01.044

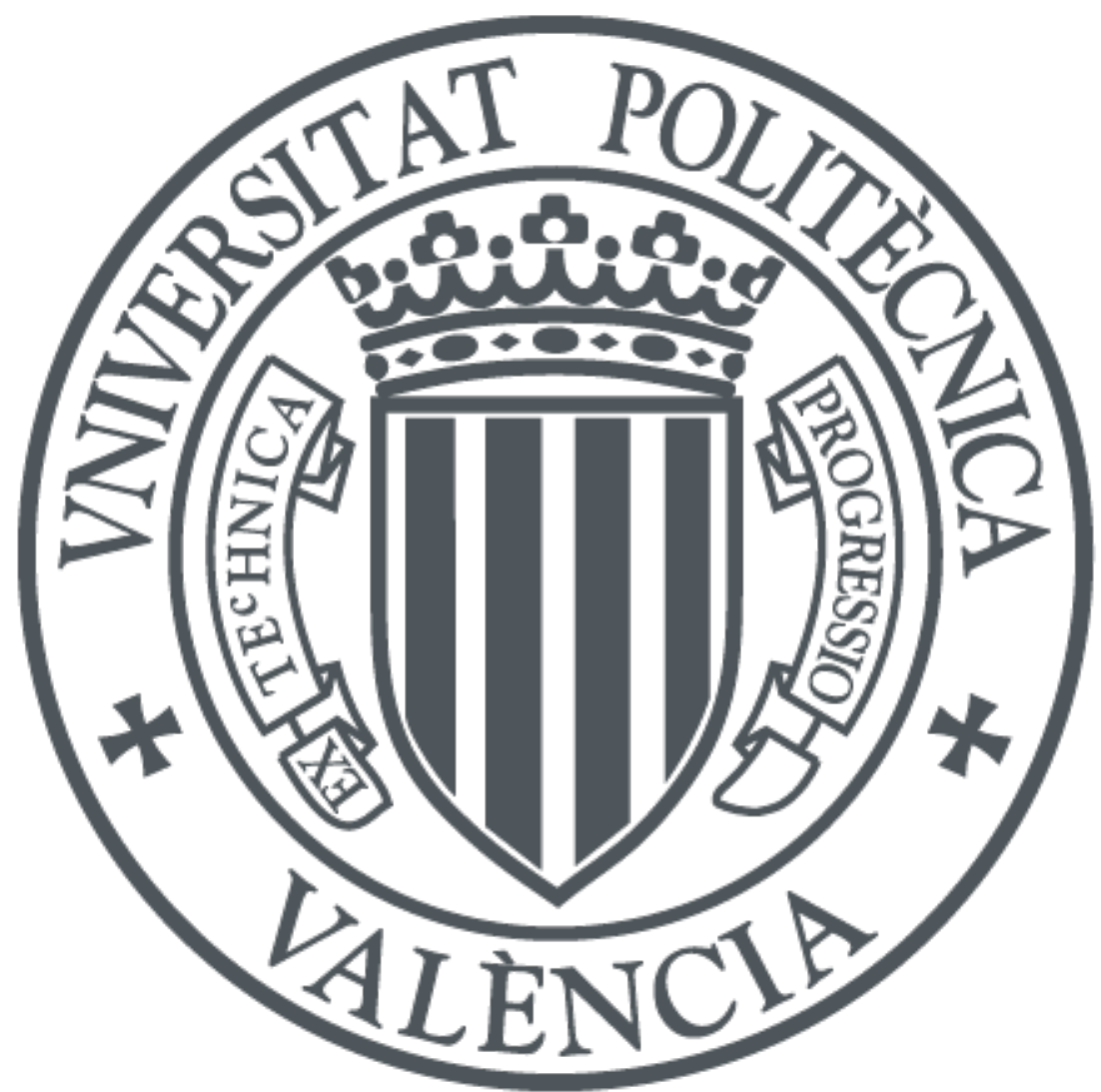

The final publication is available at

https://doi.org/10.1016/j.future.2019.01.044

Copyright Elsevier

Additional Information 


\title{
Competition in data-based service provision: Nash equilibrium characterization
}

\author{
Luis Guijarro ${ }^{\mathrm{a}, *}$, Vicent Pla ${ }^{\mathrm{a}}$, Jose R. Vidal ${ }^{\mathrm{a}}$, Maurizio Naldi $^{\mathrm{b}}$ \\ ${ }^{a}$ Universitat Politècnica de València, Spain \\ ${ }^{b}$ Università di Roma Tor Vergata, Italy
}

\begin{abstract}
This paper analyses the economic feasibility of a market for data-based services, which is a scenario envisioned in sectors that are adopting Internet of Things and/or Big Data technologies. A business model for the provision of a data-based service is proposed. In this model, service providers build services from data that is collected and sold by data providers in a market, and provide services to final users. Service providers compete strategically against each other in the data market and in the service market. The strategic interaction between the service providers is analyzed by means of non-cooperative game theory. A thorough mathematical analysis of the Nash equilibria is carried out and existence and uniqueness conditions are proved and discussed.

We conclude that if the sensitivity of users to the data-rate-to-price ratio is above the unity and the number of service provider does not exceed a given limit, a unique and meaningful equilibrium exists. We also conclude that the intensity of competition is beneficial for the users and detrimental for the service providers, while for the data providers, maximum surplus is obtained in an intermediate intensity of competition. Finally, an increase in the users' sensitivity to the data-rate-to-price ratio causes a shift on the surplus from both the service providers and data providers to the users.
\end{abstract}

Keywords: Game theory, Nash equilibrium, service provision, data providers, competition

\section{Introduction}

According to a report commissioned by the European Commission [1], the European (EU28) data market was estimated at EUR59,539 million in 2016 exhibiting a solid year-on-year growth (YoY) of 9.5\%. This report also estimated the US data market (EUR129,173 million; 11.8\% YoY), the Brazilian data market (EUR6,049 million and 14.7\% YoY) and the Japanese data market (EUR25,5 million and 6.2\% YoY). This positive trend would continue throughout the next four years. This optimistic forecasting allows us to envision the emergence of a market for data-based services, specially in sectors that are adopting Internet of Things (IoT) and/or Big Data technologies. Those companies that drive the growth for IoT devices will have the greatest potential to monetize and will likely be the first adopters of IoT data marketplaces. Examples of such industries are industrial devices (24.4\% revenue CAGR), medical devices (20.8\% revenue CAGR) and automotive/transportation devices (21.4\% revenue CAGR) [2].

Currently, many companies underutilize most of the data that they collect for internal operation purposes [3]. It is then advocated that these companies offer the data on data marketplaces as a way to monetize it [4]. However, new players that buy the data and add value to the data are needed in order to provide useful services to users. There is also interest from the institutions in removing the hurdles that hinder

\footnotetext{
* Corresponding author

Email addresses: lguijar@dcom.upv.es (Luis Guijarro), vpla@upv.es (Vicent Pla), jrvidal@upv.es (Jose R. Vidal), maurizio.naldi@uniroma2.it (Maurizio Naldi)
} 
an efficient provision of data-intensive services. For example, the European Commission has put forward legislative measures that help realize a common data space in the EU [5].

Our work focuses on the analysis of the profitability for such new players, which provide added-value services based on data. For this purpose we propose and analyze a model of the relations between all relevant players that intervene in a market with the above characteristics. The analysis applies concepts from microeconomics in the formulation of the model for the data providers, and from discrete-choice analysis in modeling the users. Game theory is thoroughly used in the paper and specifically in modeling and analyzing the competition between the service providers.

The main contributions of this paper are the following:

- A business model for the provision of a data-based service is proposed and the interaction between the different actors (data providers, service providers and users) is analyzed.

- A thorough mathematical analysis of the Nash equilibria is carried out and existence and uniqueness conditions are proved.

- It is proven that if the sensitivity of users to the data-rate-to-price ratio is above 1 and the number of service providers does not exceed a given limit, a unique and meaningful equilibrium exists.

- The surpluses at the equilibrium for the different actors are computed and discussed.

Game theory is a well-known discipline of mathematics, and its application to the modeling and analysis of the interactions in microeconomics is long-standing (see, e.g., the classical work by Von Neumann and Morgenstern [6], and the more recent book by Vives [7]). There is, however, a relatively recent trend in computer networks engineering to incorporate game theory-based models in order to take into account either the selfish behavior of the devices, either terminals or servers (see, e.g., the work on selfish behavior in CSMA/CA by Cagalj et al. [8], and the work on strategic sharing of resources by Johari and Tsitsiklis [9]) or the economic incentives of the agents, either users or providers (see, e.g., the work on internet interconnection by Laffont et al. [10], and the work on competition between Internet Service Providers by Shakkottai and Srikant [11]). Our work belongs to this trend, and it shares this feature with some of the works referred in the next subsection.

The structure of this paper is as follows. Next subsection reviews the related work. The model is described in Section 2. In Section 3, the existence and uniqueness conditions of Nash equilibria are determined and the equilibrium strategies are found. Numerical results are discussed in Section 4 to illustrate the analysis. Finally, conclusions are drawn in Section 5.

\subsection{Related work}

Reference [12] proposes a sensing-as-a-service model for smart cities supported by the Internet of Things. Specifically, it describes the role of an "extended service provider" that intermediates between sensor owners/ publishers and sensor data consumers in three different scenarios: waste management, smart agriculture and environmental management. The extended service provider builds value added services from data procured from sensors/ sensor owners/ publishers and provides them to the sensor data consumers. These roles are also modeled in our work. This sensing-as-a-service model evolves beyond other service models [13] that assume that sensing data is available per se and no incentive is required for the data providers to supply the data needed for enhanced service provision.

A business model related to ours is studied in [14], where the authors analyze a spectrum sensing service that is built using spectrum monitoring data in a cognitive radio network and that is provided to secondary users, i.e., unlicensed users that dynamically access the spectrum. Our model, however, is more general and is not dependent on technology-specific choices.

Data-based services are, however, not necessarily linked to IoT, as the emergence of Data-as-a-Service (DAAS) shows [15]. There are commercial data marketplaces already in operation, e.g. BDEX (bigdataexchange.com) for consumer data. Still, as pointed above, in order that the whole ecosystem becomes economically feasible, service providers should leverage these marketplaces for service building and provision. 
Within the scope of data-based services, there are few papers that address a formal analysis. A subscription-based big data market model is considered in [16]. An auction-based intermediation model for big data markets is modeled and analyzed in [17]. In both works, a service provider operates monopolistically in the market, and the data collector/source is modeled as an individual agent. In our work, we consider a more realistic scenario which incorporates: (i) several service providers competing strategically against each other, and (ii) a large number of data providers.

There are also some economical analyses of service provision that rely on other types of resources. Reference [18] proposes and analyses a business model for service provisioning in cloud systems. There exists competition between service providers for attracting users and also for procuring themselves with virtual resources from data centers. There are some similarities between this setting and ours, but there is not a direct equivalence between storage/computing resources and data. Within the same context of cloud services, there is a coherent and interesting set of previous works, namely [19] [20] [21] [22], which applies game theory for the analysis of the economic incentives of the different agents and the strategic interaction between the agents. Specifically, in [19], [20], and [21], the cloud users request service in advance for a set of future time slots and the service is modeled by means of $\mathrm{M} / \mathrm{M} / \mathrm{m}$ queues. In all of them, the proof of the existence of the Nash equilibrium is provided, as in our work, and additionally iterative algorithms are proposed to compute the equilibria with minimum information exchange between the players, an issue that is not covered in our work. Reference [19] analyses the competition between $n$ cloud users for the service of one cloud provider. The users are the players and their strategy is the demand rate, and the provider charges according to a polynomial request price model. Reference [20] analyses a similar scenario, but the cloud provider is now an active player, specifically the leader in a Stackelberg game, and it selects a subset of heterogeneous servers for providing service. An energy cost model is proposed for the cloud provider. And a generalized Nash equilibrium problem is stated and solved. Finally, Reference [21] analyses again the competition between $n$ cloud users for the service of one cloud provider, but each user bids now strategically for the reserved time slots. The provider allocates a number of servers following a proportional fairness criterion. There are interesting similarities and differences between [19], [20], and [21] and our work. In all these three references, the game is formulated for the cloud users, whereas in our work it is done for the service providers. While the cloud users try to maximize their own utility, the service providers try to maximize their respective profit through simultaneously competing for providing service to final users; these final users are absent in the three references. Additionally, the one cloud provider is a player in [20], but not in [19] and [21], whereas in our work the data providers are many and they are not players. Still, we provide a rationale for a cost model for the data providers, as it is done in [20].

Finally, we discussed an intermediation-based business model in a competition scenario in [23], where service providers intermediate between Wireless Sensor Networks and users. The present paper generalizes the applicability of the model beyond this specific technology setup and it provides a more detailed analytical derivation. Specifically, the equilibrium existence and uniqueness conditions of the game between the service providers are derived and discussed in this paper.

\section{Model description}

We consider a scenario where the following stakeholders act (a summary of this paper notation is given in Appendix A):

- $N$ data providers (DPs);

- $K$ service providers (SPs);

- $M$ users (Us).

These stakeholders interact through both data relationships and commercial relationships: data relationships describe how data is transferred between the different stakeholders, from the data originators (DPs) to the final data consumers (Us); and the commercial relationships describe how money is exchanged as a remuneration for transferring and processing the data. A schematic of the relationships between each class 


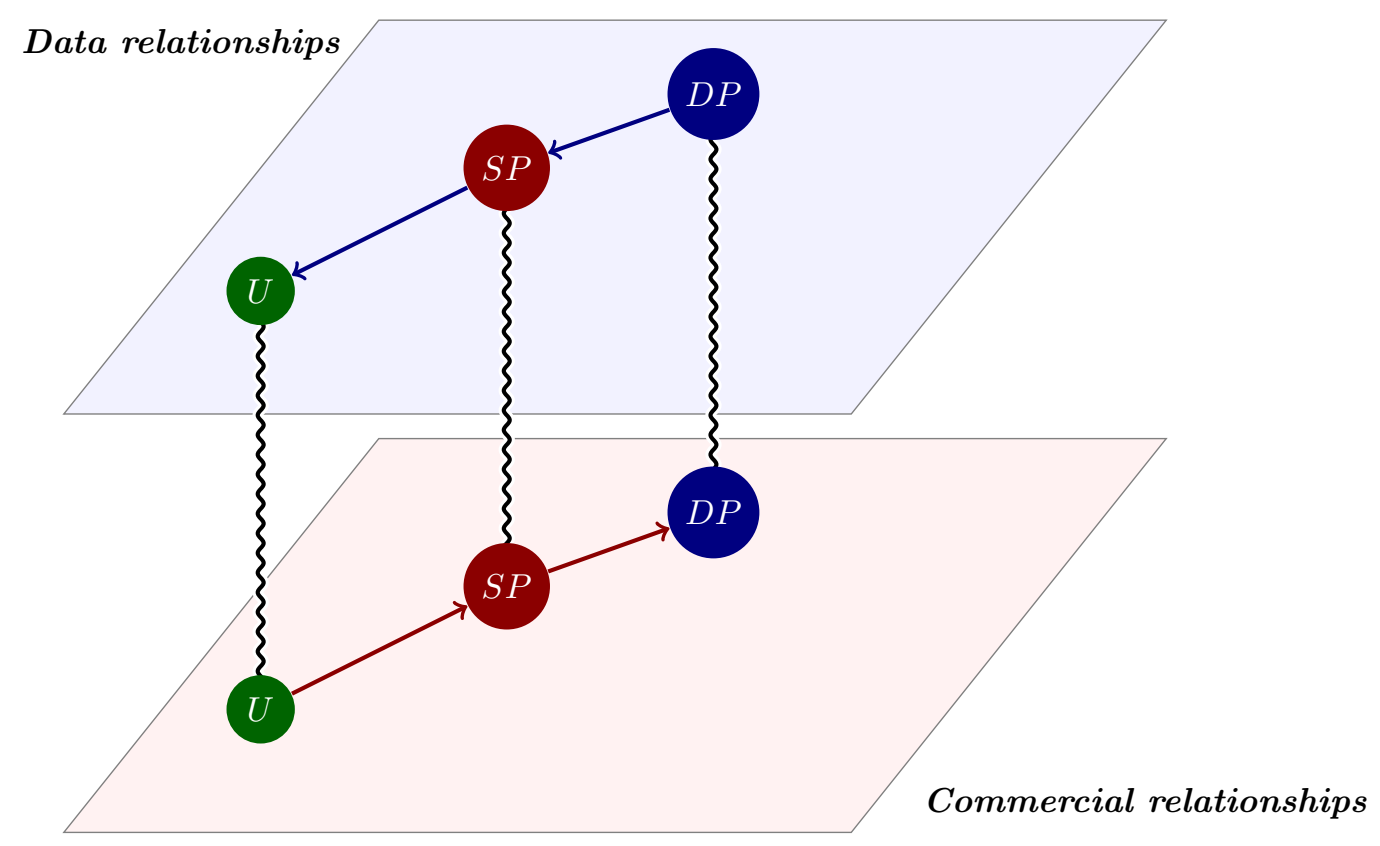

Fig. 1: Relationships between the stakeholders in service provisioning

of stakeholders is shown in Fig. 1, where the data relationships represent a transfer of data (either raw data, from the DPs to the SPs, or processed data, from the SPs to the users). As specified above, there are several instances of each class in our model.

\subsection{The data path}

Each DP may supply information of any kind that in general terms we will call 'data'. The DPs collect data as a result of their operations. DP $j$ generates data at a rate $r_{j}$, which is measured in net bits per second (nbps); this rate measures the useful data only, regardless of its physical rate or its format. Each DP can only decide whether to confer its data to the SPs or not. The specifics of the data delivery between the DPs and the SPs are not relevant to the model. Anyway, the data shall not be reused or shared by more than one SP.

The data are then bundled by the SPs. Each SP processes the data collected from the DPs to compose a service with added value and to deliver these service to the users through the network. SP $i$ uses data at the aggregate rate $R_{i}$. Each user may decide to choose the services of one SP; if it does, it will not join the other SPs; its choice is represented by the SP's index $i=1, \ldots, K$, while the value $i=0$ represents the "no-provider" option.

\subsection{The commercial path}

The commercial relationships among the stakeholders are described in the bottom plane of Fig. 1. The arrows indicate the direction of money (i.e., the reverse direction of services).

We assume that payments for services are carried out according to the following payment scheme: linear pricing for the DPs-SPs relationship, where the price driver is the data rate and the unit price is $a$, and a flat rate pricing for the SPs-users relationship.

DPs may decide whether to confer their data to the SPs or not, so that the actual number of DPs joining the SPs' platform (i.e., the number of connected DPs) is $n \leq N$. Similarly, users may decide whether to subscribe to the service. We indicate the number of user who subscribe to the services of SP $i$ as $m_{i} \leq M$. Overall, we have $\sum_{i=0}^{K} m_{i}=M$ and hence $\sum_{i=1}^{K} m_{i}=M-m_{0} \leq M$. 
The decisions taken by the DPs and the users are driven respectively by their profit and their utility: the DPs will deliver their data if it is profitable, and the users will join the SP (if any) delivering the highest utility.

Pricing has therefore an impact on both the penetration ratios (i.e., the number of stakeholders joining the market) and the profits obtained by the stakeholders. We consider first the penetration ratios and then the profits.

\subsection{Penetration ratios}

Let us examine first the decision taken by the DPs. We recall that each DP obtains a profit from the sale of its data to the SPs, and has a cost for accessing the network, so that the profit of a generic DP is

$$
\Pi_{j}=a \cdot r_{j}-f
$$

where the constant $a$ is the price per data-rate unit, which is assumed to be common to all DPs, while $f$ is the cost borne by the DP (e.g., for network payments). Both payments are expressed in a generic currency unit, which we denote by cu.

The number of DPs is assumed to be sufficiently high, and this allows us to assume that each one operates as a price-taker - the unit price $a$ and the fee $f$ are therefore given. A DP cannot influence the price $a$, either individually or as a whole, through any mechanism (e.g., bargaining, provider selection, ... ). Actually, each $\mathrm{DP}$ is invested with a limited decision power, which is to accept the price set by the market for data (where the SPs are the buyers and the DPs are the sellers) or to refuse them. And each DP's decision is made on the basis of the profit. A generic DP $j$ enters the market only if its would-be profit is non negative, i.e. $\Pi_{j} \geq 0$.

Though data supplied by the DPs is a homogeneous product, each DP supplies the data with a different data rate (e.g., it could be constrained by technological reasons). In order to model the individual differences in the data rate, we assume that the rates $r_{j}(j=1, \ldots, N)$ form a set of i.i.d. random variables following a uniform distribution over the interval $\left[0, r_{\max }\right]$.

DP $j$ will enter the market if $\Pi_{j} \geq 0$. This event has a probability

$$
\mathrm{P}\left(a r_{i}-f \geq 0\right)=1-\Psi\left(\frac{f}{a r_{\max }}\right)=1-\Psi\left(\frac{f_{m}}{a}\right),
$$

where we have defined a normalized fee $f_{m} \equiv f / r_{\max }$, and $\Psi(\cdot)$ is the CDF of the uniform distribution in $[0,1]$.

As a result of the decision process, the number of connected DPs turns out to be a random variable. Let $\mathcal{N}$ be this random variable and $n$ its expected value

$$
n \equiv \mathbb{E}[\mathcal{N}]=N\left(1-\Psi\left(\frac{f_{m}}{a}\right)\right),
$$

which is linearly decreasing with the fee $f_{m}$ in $(0, a)$.

All connected DPs will then supply an expected aggregate data rate $r_{T}$ equal to

$$
\begin{aligned}
r_{T} & \equiv \sum_{i=1}^{n} \mathbb{E}\left[r_{i} \mid r_{i} \geq \frac{f}{a}\right]=n \frac{r_{\max }}{2}\left(1+\Psi\left(\frac{f_{m}}{a}\right)\right) \\
& =\frac{N r_{\max }}{2}\left(1-\Psi^{2}\left(\frac{f_{m}}{a}\right)\right)=r_{\mathrm{off}}\left(1-\Psi^{2}\left(\frac{f_{m}}{a}\right)\right),
\end{aligned}
$$

where we have defined the maximum offered data rate as

$$
r_{\text {off }} \equiv N r_{\max } / 2 .
$$


By inverting (4) in $r_{T} \in\left(0, r_{\text {off }}\right)$, we can express the unit price $a$ as a function of the aggregate data rate $r_{T}:$

$$
a=\frac{f_{m}}{\sqrt{1-\frac{r_{T}}{r_{\mathrm{off}}}}} .
$$

This expression gives the unit price that should be paid to the DPs to deliver an aggregate data rate $r_{T}$.

Let us examine now the users' decisions. The user consumption that is expected is a discrete choice, i.e. that the user subscribes service from the SP that provides him/her with the highest utility. This is an appropriate model for services that share a basic set of characteristics, so that the services provided by the different SPs cannot be regarded as complementary; therefore, there is no incentive for a user to bundle a combined service offer from more than one SP. Despite this similarity, the services are not identical, since they are differentiated by an additional service feature apart from the price; this feature is the data rate acquired by the SPs for the service provision. We adopt the discrete-choice model described in [24]. In this model, a user $u$ may choose an option in a discrete set; its utility when choosing option $i$ is $v_{i}+\kappa_{u, i}$. This utility is made up of two terms, representing respectively the objective characteristics of option $i\left(v_{i}\right)$ and the unobserved user-specific part $\left(\kappa_{u, i}\right)$, which is considered to be a random variable. The probability of a user choosing option $i$ can be obtained from the distribution of the variables $\kappa_{u, i}$, and this can be taken as the fraction of the users that make that choice, as the user population size $M$ gets larger.

Each SP $i$ 's subscriber is charged a flat-rate price $p_{i}$ and receives a service composed from an aggregate data rate $R_{i}$. In this case, the objective part $v_{i}$ is proposed to be [25]

$$
v_{i}=\mu \log \left(\frac{R_{i}}{p_{i}}\right)
$$

for which we can observe what follows:

1. the user's utility grows with the service data rate;

2. the dependence on $R_{i}$ is logarithmic, since user experience and satisfaction in resource-based services follow logarithmic laws [26];

3. a similar reasoning can be applied to $1 / p_{i}$, which lead us to the proposition that the ratio $R_{i} / p_{i}$ should be the relevant magnitude;

4. $\mu>0$ acts as a sensitivity parameter;

5. the ratio $R_{0} / p_{0}$ corresponds the "no-provider" option, and its value describes the appeal of such option to the user.

As regards the unobserved user-specific part of the utility, each $\kappa_{u, i}$ is proposed to be independently and identically Gumbel-distributed with mean 0 and parameter $\nu$.

Besides, for sufficiently high $M$, the users can be realistically assumed to be price-takers.

Each user may therefore choose either to subscribe to any of the $K$ providers or give up the service (the "no-provider" option). For the generic $i^{t h}$ SP we can therefore introduce the penetration ratio $\rho_{i}$, i.e. the ratio of the number $m_{i}$ of users who subscribe to the $i^{t h}$ service to the total number of users $M$. Under the above assumptions, it can be shown [27] that

$$
\rho_{i}=\frac{m_{i}}{M}=\frac{\left(R_{i} / p_{i}\right)^{\alpha}}{\sum_{l=0}^{K}\left(R_{l} / p_{l}\right)^{\alpha}}, \quad i=1, \ldots, K,
$$

where $\alpha=\mu / \nu$ is a parameter that represents the sensitivity of users to the data-rate-to-price ratio. The higher the value of $\alpha$ the more attractive to users will be those with a high ratio. In the limit, if the sensitivity was infinite, all users will subscribe to the SP with the highest ratio. Conversely, with a null sensitivity users will distribute equally among the $K$ SPs plus the "no-provider". 


\subsection{Competition game}

In this subsection, the competition between the SPs is described and its modeling as a game is justified.

There is a competition among the SPs to get the data from the DPs. The DPs are considered as pricetakers and are described by the inverse supply function (6). Each SP can instead influence the data price both directly, through its own choice of $R_{i}$, and strategically, through each competitor's choice $R_{l}, l \neq i$. The interaction between the SPs can be studied as a non-cooperative simultaneous game, where each SP chooses the data rate $R_{i}$ to be procured, getting a payoff $-a \cdot R_{i}$, where $a$ is given by (6). We assume that the data procured from the DPs cannot be reused or shared among the SPs. Then, the aggregate data rate $r_{T}$ will match the sum of the SPs' requests, that is, $r_{T}=\sum_{j=1}^{K} R_{j}$, and therefore, this provides the basis for hereafter specifying $a$ as a function of $\sum_{j=1}^{K} R_{j}$.

Note that the assumption that each unit of data can only be sold once and that the data purchased by an SP cannot be shared with the other SPs corresponds to a situation in which each unit of data is sold to an SP, and hence this SP holds the exclusive rights to exploit it. In a sense, this may be regarded as if the SPs were leasing from the DPs a fraction of the data acquisition capability. This is an appropriate model where the data supplied by the DPs are considered a raw material that is procured by the SPs, and each SP purchases a certain amount of that raw material to process it and produce a service with an added value.

The $K$ SPs not only compete for the DPs' data, but also for the users, whose demand is modeled as described above. The two competitions are proposed to be executed simultaneously rather than sequentially. The logic is that the selection of $R_{i}$ should be seen as an operational expense, and not an investment choice. As an operational expense, the choices of $R_{i}$ and of the price take place at the same time scale.

Thus, in the combined game, the players are the $k$ SPs. SP $i$ 's strategy is the pair $p_{i}$ and $R_{i}$; and SP $i$ 's payoff is given by

$$
\Pi_{i}=p_{i} M \rho_{i}-a\left(\sum_{j=1}^{K} R_{j}\right) R_{i}, \quad i=1, \ldots, K,
$$

where $\rho_{i}$ and $a(\cdot)$ are given by (8) and (6), respectively.

\subsection{Profits}

As stated before, the decisions taken by the DPs to release their data and by the users to subscribe to the service are respectively driven by their profit and their utility. At the same time the SPs acquire their resources and price their services so as to maximize their profit. Profits (and utilities) are therefore essential to define the actual configuration of the market, represented by the actual number of users, DPs, and SPs that make up the market. In this subsection we compute the aggregated profits of each group of stakeholders.

We start with the aggregate profit of the DPs, defined as the sum of all (connected) DPs' profits

$$
\Pi_{\mathrm{DPs}}=\mathbb{E}\left[\sum_{j=1}^{N} \Pi_{j}\right]=\sum_{i=1}^{N} \mathbb{E}\left[\Pi \mid r_{i} \geq \frac{f}{a}\right] \mathrm{P}\left(r_{i} \geq \frac{f}{a}\right)=r_{\mathrm{off}} a\left(1-\frac{f_{m}}{a}\right)^{2},
$$

where the unit price $a$ is related to the aggregate data rate $r_{T}$ through Equation (6).

As to the users, we can similarly define their surplus as their aggregate utility, i.e., the sum of all subscribers' utilities. Following [27], we have

$$
\Pi_{\mathrm{users}}=\sum_{u=1}^{M} \mathbb{E}\left[\max _{i}\left\{v_{i}+\kappa_{u, i}\right\}\right] .
$$

When all SPs provide the same data rate and charge the same price $\left(R_{i}=R\right.$ and $p_{i}=p$ for $\left.i=1,2, \ldots K\right)$, which we will refer to as the symmetric case, the user surplus is

$$
\Pi_{\mathrm{users}}^{(s)}=M \log \left[\left(\frac{R_{0}}{p_{0}}\right)^{\alpha}+K\left(\frac{R}{p}\right)^{\alpha}\right],
$$


and the overall profit of the SPs is

$$
\Pi_{\mathrm{SPs}}^{(s)}=K\left(p M \frac{\left(\frac{R}{p}\right)^{\alpha}}{\left(\frac{R_{0}}{p_{0}}\right)^{\alpha}+K\left(\frac{R}{p}\right)^{\alpha}}-a(K R) R\right) .
$$

\section{Analysis}

In the previous section, a non-cooperative game between the the SPs was defined. Here we analyze the game using the solution concept of Nash equilibrium.

We establish conditions for existence and uniqueness of equilibria and find the corresponding strategic profiles. We show that all equilibrium points are symmetric in the sense that all SPs that enter the market play the same strategy. In particular, it is proven that if the users' sensitivity is below the unity $(\alpha<1)$ no equilibrium exists; and that if it is above the unity $(\alpha>1)$ and the total number of SPs does not exceed a given limit $\left(K<\eta_{M}\right)$ there is a unique equilibrium.

Next we introduce some additional notation and definitions, and then the analysis is presented through a number of propositions and corollaries. Besides, auxiliary lemmas that are used in the proofs of the propositions are included in Appendix B.

\subsection{Introductory remarks}

From (8) and (9), the profit of SP $i$ can be written as

$$
\Pi_{i}\left(p_{i}, R_{i} ; \boldsymbol{p}_{-i}, \boldsymbol{R}_{-i}\right)=M p_{i} \frac{\left(R_{i} / p_{i}\right)^{\alpha}}{\sum_{j=0}^{K}\left(R_{j} / p_{j}\right)^{\alpha}}-a\left(\sum_{j=1}^{K} R_{j}\right) R_{i}, \quad i=1, \ldots, K,
$$

where $\boldsymbol{p}_{-i}=\left(p_{1}, \ldots, p_{i-1}, p_{i+1}, \ldots, p_{K}\right)$ and $\boldsymbol{R}_{-i}=\left(R_{1}, \ldots, R_{i-1}, R_{i+1}, \ldots, R_{K}\right)$. If it does not lead to ambiguity, we will also use the simpler notation $\Pi_{i}\left(p_{i}, R_{i}\right)$ or $\Pi_{i}$ to refer to $\Pi_{i}\left(p_{i}, R_{i} ; \boldsymbol{p}_{-i}, \boldsymbol{R}_{-i}\right)$.

It can be observed that the profits $\Pi_{i}$ 's depend on $R_{0} / p_{0}$, but not on the individual values of $p_{0}$ and $R_{0}$. Hence the number of parameters can be reduced by one by setting $R_{0}=1$. Additionally, we can normalize by $p_{0}$ all prices, profits and utilities, which boils down to setting $p_{0}=1$.

We use the strategy $\left(p_{l}, R_{l}\right)=(0,0)$ to capture the situation where SP $l$ does not enter the market. This convention requires some technical changes in the definition of $\Pi_{l}$ (see (14)) to include the cases in which there are some $p_{l}=0, l=1, \ldots, K$. These changes take into account the following:

- When an SP $l$ does not enter the market its profit is zero, that is $\Pi_{l}(0,0)=0$, and for the profit of the rest of SPs it is as if SP $l$ did not exist.

- According to (14), $\Pi_{l}\left(p_{l}, 0\right)=0$ for all $p_{l}>0$. Moreover, from (8), if $R_{l}=0$ no users will subscribe to SP $l$ and, hence, the actual price $p_{l}$ is not relevant. Thus, we can take all the strategies of the form $\left(p_{l}, 0\right)$, with $p_{l}>0$, out of the domain of $\Pi_{l}\left(p_{l}, 0\right)$ and consider all of them to be represented by the strategy $(0,0)$.

- The strategies of the form $\left(0, R_{l}\right)$ with $R_{l}>0$ yield a lower profit that the strategy $(0,0)$ to SP $l$. Therefore, a strategy of that form could not constitute an equilibrium point.

Based on these observations, we propose the following definition and proposition, where the latter provides necessary conditions for an equilibrium point. The equilibrium concept used in this paper is the Nash equilibrium, which is a strategy profile where each player has no incentive to deviate from its strategy provided that the other players use their respective equilibrium strategies.

Let $\left(\boldsymbol{p}_{-l}, \boldsymbol{R}_{-l}\right)$ denote a pair of $(K-1)$-tuples such that

$$
\left(p_{i}, R_{i}\right) \in\{(0, \infty) \times(0, \infty)\} \cup\{(0,0)\}, \quad i=1, \ldots, l-1, l+1, \ldots, K,
$$


and $R_{1}+\cdots+R_{l-1}+R_{l+1}+\cdots+R_{K}<r_{\text {off }}$. We define the function for the profit of SP $l, \Pi_{l}\left(p_{l}, R_{l} ; \boldsymbol{p}_{-l}, \boldsymbol{R}_{-l}\right)$, as follows

$$
\Pi_{i}\left(p_{i}, R_{i} ; \boldsymbol{p}_{-i}, \boldsymbol{R}_{-i}\right)= \begin{cases}0 & \text { if } p_{i}=0, \\ M p_{i} \frac{\left(R_{i} / p_{i}\right)^{\alpha}}{1+\sum_{\substack{j=1 \\ p_{j} \neq 0}}^{K}\left(R_{j} / p_{j}\right)^{\alpha}}-a\left(\sum_{j=1}^{K} R_{j}\right) R_{i} & \text { otherwise, }\end{cases}
$$

in the domain

$$
\mathcal{D}_{i}\left(\boldsymbol{R}_{-i}\right)=\{(0,0)\} \cup\left\{(0, \infty) \times\left(0, r_{\text {off }}-\sum_{j \neq i} R_{j}\right)\right\} .
$$

Proposition 1. Let $\left(\boldsymbol{p}^{*}, \boldsymbol{R}^{*}\right)=\left(\left(p_{1}^{*}, \ldots, p_{K}^{*}\right),\left(R_{1}^{*}, \ldots, R_{K}^{*}\right)\right)$ be a Nash equilibrium. Then, the strategy of each $S P l$ (with $l=1, \ldots, K)$ must satisfy one of the following:

1. SP l will not join the market: $\left(p_{l}^{*}, R_{l}^{*}\right)=(0,0)$;

2. The profit of $S P l$ has a maximum at $\left(p_{l}^{*}, R_{l}^{*}\right)$ and, hence,

$$
\frac{\partial \Pi_{l}}{\partial p_{l}}\left(p_{l}^{*}, R_{l}^{*} ; \boldsymbol{p}_{-l}, \boldsymbol{R}_{-l}\right)=\frac{\partial \Pi_{l}}{\partial R_{l}}\left(p_{l}^{*}, R_{l}^{*} ; \boldsymbol{p}_{-l}, \boldsymbol{R}_{-l}\right)=0 .
$$

\subsection{Nash equilibrium with no SPs entering the market}

The next proposition gives necessary and sufficient conditions for $(\mathbf{0}, \mathbf{0})$ to be a Nash equilibrium ${ }^{1}$. The strategic profile corresponds to the case in which none of the $K$ SPs enter the market.

Proposition 2. 1. When $0 \leq \alpha<1,(\mathbf{0}, \mathbf{0})$ cannot be an equilibrium.

2. When $\alpha=1,(\mathbf{0}, \mathbf{0})$ is an equilibrium if and only if $f_{m} / M \geq 1$.

3. When $\alpha>1,(\mathbf{0}, \mathbf{0})$ is an equilibrium if and only if $f_{m} / M \geq \frac{(\alpha-1)^{1-1 / \alpha}}{\alpha}$.

(a) If $f_{m} / M<1 / 2,(\mathbf{0}, \mathbf{0})$ is not an equilibrium.

(b) If $1 / 2 \leq f_{m} / M<1,(\mathbf{0}, \mathbf{0})$ is an equilibrium if, and only if, $\alpha \in\left[\alpha_{1}, \alpha_{2}\right]$, where $1<\alpha_{1} \leq 2 \leq \alpha_{2}$.

(c) If $f_{m} / M \geq 1,(\mathbf{0}, \mathbf{0})$ is an equilibrium.

Proof. For $(\mathbf{0}, \mathbf{0})$ to be an equilibrium it is required that

$$
\Pi_{i}\left(p_{i}, R_{i} ; \mathbf{0}, \mathbf{0}\right)=M p_{i} \frac{\left(R_{i} / p_{i}\right)^{\alpha}}{1+\left(R_{i} / p_{i}\right)^{\alpha}}-f_{m} \frac{R_{i}}{\sqrt{1-R_{i} / r_{\mathrm{off}}}} \leq \Pi_{i}(0,0 ; \mathbf{0}, \mathbf{0})=0,
$$

for all $\left(p_{i}, R_{i}\right) \in \mathcal{D}_{i}\left(\boldsymbol{R}_{-i}\right)$. A simple algebraic manipulation of (19) leads to the equivalent condition

$$
\frac{\left(R_{i} / p_{i}\right)^{\alpha-1}}{1+\left(R_{i} / p_{i}\right)^{\alpha}} \leq \frac{f_{m} / M}{\sqrt{1-R_{i} / r_{\mathrm{off}}}}, \quad \forall\left(p_{i}, R_{i}\right) \in \mathcal{D}_{i}\left(\boldsymbol{R}_{-i}\right)
$$

which is equivalent to

$$
\sup _{R_{i} / p_{i}>0} \frac{\left(R_{i} / p_{i}\right)^{\alpha-1}}{1+\left(R_{i} / p_{i}\right)^{\alpha}} \leq \inf _{R_{i} / r_{\text {off }}>0} \frac{f_{m} / M}{\sqrt{1-R_{i} / r_{\text {off }}}}=\frac{f_{m}}{M},
$$

where the equality follows by application of Lemma 1 in Appendix B.

The proof is now completed by obtaining the value of the supremum on the left hand side of (21). For this we need to consider the three cases in the statement of the proposition.

\footnotetext{
${ }^{1} \mathbf{0}$ (respectively, 1) denotes a vector of the appropriate dimension whose entries are all zero (respectively, one).
} 


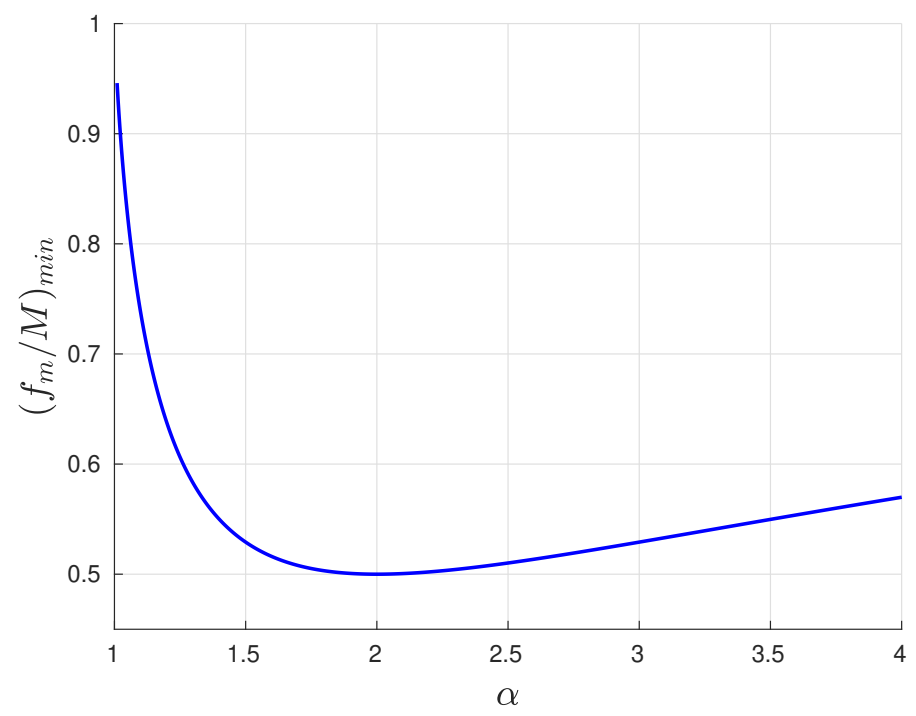

Fig. 2: Minimum value of $f_{m} / M$ for which $(\mathbf{0}, \mathbf{0})$ is an equilibrium.

1. If $0 \leq \alpha<1$,

$$
\sup _{R_{i} / p_{i}>0} \frac{1}{\left(1+\left(R_{i} / p_{i}\right)^{\alpha}\right)\left(R_{i} / p_{i}\right)^{1-\alpha}}=\infty
$$

2. If $\alpha=1$,

$$
\sup _{R_{i} / p_{i}>0} \frac{1}{1+R_{i} / p_{i}}=1
$$

3. If $\alpha>1$, by application of Lemma 2 in Appendix B,

$$
\sup _{R_{i} / p_{i}>0} \frac{\left(R_{i} / p_{i}\right)^{\alpha-1}}{1+\left(R_{i} / p_{i}\right)^{\alpha}}=\frac{1}{\alpha}(\alpha-1)^{1-1 / \alpha} .
$$

Now the three subcases in case 3 follow as an immediate application of Lemma 3 in Appendix B.

The results of Proposition 2 are illustrated in Fig. 2, the minimum value of $f_{m} / M$ for which $(\mathbf{0}, \mathbf{0})$ is an equilibrium is plotted as a function of $\alpha>1$.

For each value of $\alpha$, if the fee paid by the DPs to the network access provider (normalized by the maximum information rate $r_{\max }$ and by the market size $\left.M\right)$ is at least $\left(f_{m} / M\right)_{\min }$, there will be an equilibrium in which no SPs enter the market. Furthermore, it is worth noting that the value of $\left(f_{m} / M\right)_{\text {min }}$ only depend on the sensitivity parameter $\alpha$.

\subsection{Nash equilibria where some SPs enter the market}

Now, equilibrium points where at least an SP enters the market are considered. To this end, we first seek strategies $\left(p_{i}, R_{i}\right) \neq(0,0)$ that satisfy the second condition of Proposition 1.

The derivatives of $\rho_{i}$ in (8) are given as

$$
\begin{aligned}
\frac{\partial \rho_{i}}{\partial p_{i}} & =-\frac{\alpha}{p_{i}} \rho_{i}\left(1-\rho_{i}\right), \\
\frac{\partial \rho_{i}}{\partial R_{i}} & =\frac{\alpha}{R_{i}} \rho_{i}\left(1-\rho_{i}\right),
\end{aligned}
$$


and using these we obtain the partial derivatives of $\Pi_{i}$

$$
\begin{aligned}
& \frac{\partial \Pi_{i}}{\partial p_{i}}=M \rho_{i}\left(1-\alpha\left(1-\rho_{i}\right)\right), \\
& \frac{\partial \Pi_{i}}{\partial R_{i}}=M p_{i} \frac{\alpha}{R_{i}} \rho_{i}\left(1-\rho_{i}\right)-a\left(\sum_{j=1}^{K} R_{j}\right)-a^{\prime}\left(\sum_{j=1}^{K} R_{j}\right) R_{i},
\end{aligned}
$$

where $a(\cdot)$ is the function defined in (6).

Now we address separately the cases where $0 \leq \alpha \leq 1$ and $\alpha>1$. As it will be seen, the second is more complex and leads to a richer scenario.

Proposition 3. When $0 \leq \alpha \leq 1$ the only possible equilibrium is $(\mathbf{0}, \mathbf{0})$.

Proof. Let $\left(\boldsymbol{p}^{*}, \boldsymbol{R}^{*}\right) \neq(\mathbf{0}, \mathbf{0})$ be an equilibrium point. Then, there must exist an $i=1, \ldots, K$ such that $p_{i}^{*} \neq 0, R_{i}^{*} \neq 0$ and, by Proposition 1 , the equality (18) must hold.

Now we examine three different cases according to the value of $\alpha$, and show that in none of them it is possible to meet (18).

$\alpha=0$ :

Clearly

$$
\frac{\partial \Pi_{i}}{\partial p_{i}}=\frac{M}{1+K}>0
$$

$0<\alpha<1$ :

If $\rho_{i}>0$

$$
\frac{\partial \Pi_{i}}{\partial p_{i}}=M \rho_{i}\left(1-\alpha\left(1-\rho_{i}\right)\right)>M \rho_{i}(1-\alpha)>0 .
$$

Therefore, $\frac{\partial \Pi_{i}}{\partial p_{i}}=0$ implies that $\rho_{i}=0$, and hence $R_{i}^{*}=0$, which contradicts the initial assumption. $\alpha=1$ :

In this case

$$
\frac{\partial \Pi_{i}}{\partial p_{i}}=M \rho_{i}^{2} .
$$

Thus, $\frac{\partial \Pi_{i}}{\partial p_{i}}=0$ again leads to the same contradiction as in the previous case $\left(R_{i}^{*}=0\right)$.

A summary about the existence and characteristics of equilibria when $0 \leq \alpha \leq 1$ is provided in the following corollary, which is an immediate consequence of Proposition 2 and Proposition 3.

Corollary 1. 1. When $0 \leq \alpha<1$, no equilibrium exists.

2. When $\alpha=1$, if $f_{m} / M \geq 1$ then $(\mathbf{0}, \mathbf{0})$ is the unique equilibrium, and if $f_{m} / M<1$ then no equilibrium exists.

In contrast to the case where $\alpha \leq 1$, when $\alpha>1$ there can exist equilibrium points different from $(\mathbf{0}, \mathbf{0})$. In Proposition 4 necessary conditions for the existence and some structural properties of such equilibrium points are provided. Before formulating this proposition, some new definitions and notations need to be introduced.

Let $\kappa(\boldsymbol{p}, \boldsymbol{R})$ denote the number of SPs that enter the market at the point $(\boldsymbol{p}, \boldsymbol{R}) \neq(\mathbf{0}, \mathbf{0})$, and let $\mathcal{I}(\boldsymbol{p}, \boldsymbol{R})$ be the set of their indices, that is,

$$
\begin{aligned}
& \mathcal{I}(\boldsymbol{p}, \boldsymbol{R})=\left\{i \in\{1, \ldots, K\} \mid\left(p_{i}, R_{i}\right) \neq(0,0)\right\}, \\
& \kappa(\boldsymbol{p}, \boldsymbol{R})=|\mathcal{I}(\boldsymbol{p}, \boldsymbol{R})| .
\end{aligned}
$$


Moreover, define $A(k)$ by

$$
A(k)=\left(\frac{f_{m}}{M} \frac{\alpha}{\alpha-1}\right)^{-2}\left(\frac{\alpha}{\alpha-1}-k\right)^{2 / \alpha} .
$$

Now, when $A(k)>1$ we define $z_{0}(k)$ as the solution in $(0,1)$ of

$$
A(k)(1-x)^{3}-\left(1-\frac{x}{\beta}\right)^{2}=0
$$

where

$$
\beta=\frac{k}{k-1 / 2}>1 .
$$

Lemma 4 in Appendix B shows that (35) has exactly one solution in $(0,1)$ if $A(k)>1$. Therefore, $z_{0}(k)$ is well defined.

Proposition 4. If $\left(\boldsymbol{p}^{*}, \boldsymbol{R}^{*}\right)$ is an equilibrium different from $(\mathbf{0}, \mathbf{0})$ and $\alpha>1$, then

1. $\kappa \equiv \kappa\left(\boldsymbol{p}^{*}, \boldsymbol{R}^{*}\right)<\eta_{M}$, with $\eta_{M}$ defined by

$$
\eta_{M} \equiv \frac{\alpha}{\alpha-1}-\left(\frac{f_{m}}{M} \frac{\alpha}{\alpha-1}\right)^{\alpha}<\frac{\alpha}{\alpha-1} .
$$

2. The strategy of all SPs joining the market is the same

$$
\left(p_{i}^{*}, R_{i}^{*}\right)=\left(p^{*}(\kappa), R^{*}(\kappa)\right) \quad \forall i \in \mathcal{I}\left(\boldsymbol{p}^{*}, \boldsymbol{R}^{*}\right),
$$

with

$$
\begin{aligned}
& R^{*}(\kappa)=\frac{z_{0}(\kappa)}{\kappa} r_{o f f}, \\
& p^{*}(\kappa)=\left(\frac{\alpha}{\alpha-1}-\kappa\right)^{1 / \alpha} R^{*}(\kappa),
\end{aligned}
$$

and attract the same fraction of users

$$
\rho_{i}=1-\frac{1}{\alpha} \quad \forall i \in \mathcal{I}\left(\boldsymbol{p}^{*}, \boldsymbol{R}^{*}\right) .
$$

Proof. On account of Proposition 1 , for each $i=1, \ldots, K$, we have either $\left(p_{i}^{*}, R_{i}^{*}\right)=(0,0)$ or

$$
\frac{\partial \Pi_{i}}{\partial p_{i}}\left(p_{i}^{*}, R_{i}^{*}\right)=\frac{\partial \Pi_{i}}{\partial R_{i}}\left(p_{i}^{*}, R_{i}^{*}\right)=0 .
$$

From these and $(27)$ it follows that if $\left(p_{i}^{*}, R_{i}^{*}\right) \neq(0,0)$, then necessarily $\rho_{i}=1-1 / \alpha$.

We assume, without any loss of generality, that the SPs are numbered in such a way that

$$
\begin{aligned}
\rho_{i} & =1-\frac{1}{\alpha}, & & i=1,2, \ldots, \kappa \\
\left(p_{i}^{*}, R_{i}^{*}\right) & =(0,0), & i & =\kappa+1, \kappa+2, \ldots, K .
\end{aligned}
$$

In other words, we are saying that $\mathcal{I}(\boldsymbol{p}, \boldsymbol{R})=\{1,2, \ldots, \kappa\}$, where $\mathcal{I}(\boldsymbol{p}, \boldsymbol{R})$ is as defined in (32). From the above it easily follows that

$$
\frac{R_{i}^{*}}{p_{i}^{*}}=\left(\frac{\alpha}{\alpha-1}-\kappa\right)^{-1 / \alpha}, \quad i=1,2, \ldots, \kappa
$$


Combining $\frac{\partial \Pi_{i}}{\partial R_{i}}=0$ with $(28)$ and (41) we obtain

$$
\left(1-\frac{1}{r_{\text {off }}}\left(\sum_{j=1}^{\kappa} R_{j}^{*}-R_{i}^{*} / 2\right)\right)^{2}=A(\kappa)\left(1-\frac{1}{r_{\text {off }}} \sum_{j=1}^{\kappa} R_{j}^{*}\right)^{3}, \quad i=1,2, \ldots, \kappa,
$$

being $A(\kappa)$ as defined in (34). We note that the right hand side of the above equality does not depend on $i$. Hence

$$
\left|1-\frac{1}{r_{\mathrm{off}}}\left(\sum_{j=1}^{\kappa} R_{j}^{*}-R_{i}^{*} / 2\right)\right|=\left|1-\frac{1}{r_{\mathrm{off}}}\left(\sum_{j=1}^{\kappa} R_{j}^{*}-R_{l}^{*} / 2\right)\right|, \quad i, l=1,2, \ldots, \kappa .
$$

From this, since $\sum_{j=1}^{\kappa} R_{j}^{*}<r_{\text {off }}$, we can deduce that

$$
p_{i}^{*}=p_{l}^{*} \quad i, l=1,2, \ldots, \kappa,
$$

and, by recalling (43), that

$$
R_{i}^{*}=R_{l}^{*} \quad i, l=1,2, \ldots, \kappa .
$$

Therefore, we have shown that all the SPs entering the market play a common strategy

$$
\left(p_{i}^{*}, R_{i}^{*}\right)=\left(p^{*}(\kappa), R^{*}(\kappa)\right) \quad i=1,2, \ldots, \kappa .
$$

Let us define

$$
z(\kappa)=\frac{R^{*}(\kappa)}{r_{\text {off }} / \kappa} .
$$

By substituting $R^{*}(\kappa)=z(\kappa) r_{\text {off }} / \kappa$ into $(44)$ we obtain

$$
A(\kappa)\left((1-z(\kappa))^{3}-\left(1-\frac{z(\kappa)}{\beta}\right)^{2}=0\right.
$$

where $\beta=\kappa /(\kappa-1 / 2)$, as defined in $(36)$.

From the hypothesis of the proposition, a value $R^{*}(\kappa)$ such that $\sum_{i=1}^{\kappa} R_{i}^{*}=\kappa R^{*}(\kappa)<r_{\text {off }}$ must exist. Consequently, we must have that $A(\kappa)>1$ (see Lemma 4), which yields

$$
\kappa<\frac{\alpha}{\alpha-1}-\left(\frac{\alpha}{\alpha-1} \frac{f_{m}}{M}\right)^{\alpha} \text {. }
$$

Under this condition there exists a unique solution to $(46)$ in $(0,1)$, which is denoted as $z_{0}(\kappa)$. Hence

$$
R^{*}(\kappa)=\frac{z_{0}(\kappa)}{\kappa} r_{\text {off }},
$$

and (39) follows immediately from (43).

Proposition 4 gives necessary conditions for the existence of equilibria where some SPs join the market. It also characterizes the strategic profiles of such equilibria. However, nothing has been said about sufficient conditions that will guarantee the existence of this type of equilibria. Similarly, this proposition does not exclude the possibility that this type of equilibria could coexist with the equilibrium where no SPs join the market. This possibility is ruled out by the following corollary.

Corollary 2. If $(\mathbf{0}, \mathbf{0})$ is an equilibrium then it is the only one.

Proof. When $0 \leq \alpha \leq 1$ the result is trivial on account of Corollary 1 .

We now turn to the case $\alpha>1$. Assume that $(\mathbf{0}, \mathbf{0})$ is an equilibrium. Then, from Proposition 2 we have

$$
\frac{f_{m}}{M} \geq \frac{\alpha-1}{\alpha} \frac{1}{(\alpha-1)^{1 / \alpha}}
$$

which is easily seen to be equivalent to $\eta_{M} \leq 1$. Therefore, there cannot exist an equilibrium meeting the conditions of Proposition 4. 
The following two propositions establish sufficient conditions for the existence of equilibria in which some SPs enter the market. The first proposition considers the case where all the SPs join the market and the second one the case some SPs, but not all, join the market.

Proposition 5. Suppose that $\alpha>1$. Then, if $K<\eta_{M}$ the strategic profile in which all the $K$ SPs play the strategy $\left(p^{*}(K), R^{*}(K)\right)$ given by $(39)$ and $(38)$ is an equilibrium.

Proof. Let $\hat{\Pi}_{i}(p, R)$ be a function defined in $\mathcal{D}_{i}\left(R^{*}(K) \cdot \mathbf{1}\right)$ given as

$$
\hat{\Pi}_{i}(p, R)=\Pi_{i}\left(p, R ; p^{*}(K) \cdot \mathbf{1}, R^{*}(K) \cdot \mathbf{1}\right) .
$$

We need to prove that

$$
\hat{\Pi}_{i}(p, R) \leq \hat{\Pi}_{i}\left(p^{*}(K), R^{*}(K)\right)=\Pi_{i}\left(p^{*}(K) \cdot \mathbf{1}, R^{*}(K) \cdot \mathbf{1}\right), \quad \forall(p, R) \in \mathcal{D}_{i}\left(R^{*}(K) \cdot \mathbf{1}\right) .
$$

The point $\left(p^{*}(K), R^{*}(K)\right)$ is, by definition, a critical point of $\hat{\Pi}_{i}(p, R)$. Next, we show that the Hessian of $\hat{\Pi}_{i}(p, R)$, at $\left(p^{*}(K), R^{*}(K)\right)$, is definite negative and, in consequence, $\left(p^{*}(K), R^{*}(K)\right)$ is a local maximum. Then, we show that $\hat{\Pi}_{i}\left(p^{*}(K), R^{*}(K)\right)>0=\hat{\Pi}_{i}(0,0)$. Finally, by studying the value of $\hat{\Pi}_{i}(p, R)$ as $(p, R)$ approaches the borders of $\mathcal{D}_{i}\left(R^{*}(K) \cdot \mathbf{1}\right)$ we will prove that $\left(p^{*}(K), R^{*}(K)\right)$ is indeed a global maximum.

From $(27)$ and $(28)$, and by the same procedure used to derive them, we obtain

$$
\begin{aligned}
\frac{\partial^{2} \hat{\Pi}}{\partial p_{i}^{2}}\left(p^{*}(K), R^{*}(K)\right) & =-\frac{(\alpha-1)^{2}}{\alpha} \frac{M}{p^{*}(K)} \\
\frac{\partial^{2} \hat{\Pi}}{\partial p_{i} \partial R_{i}}\left(p^{*}(K), R^{*}(K)\right) & =\frac{(\alpha-1)^{2}}{\alpha} \frac{M}{R^{*}(K)} \\
\frac{\partial^{2} \hat{\Pi}}{\partial R_{i}^{2}}\left(p^{*}(K), R^{*}(K)\right) & =-\frac{(\alpha-1)^{2}}{\alpha} \frac{M p^{*}(K)}{\left(R^{*}(K)\right)^{2}}-2 a^{\prime}\left(r_{\text {off }} z_{0}(K)\right)-R_{i} a^{\prime \prime}\left(r_{\text {off }} z_{0}(K)\right),
\end{aligned}
$$

where we have used that $\rho_{i}\left(p^{*}(K), R^{*}(K)\right)=1-1 / \alpha$ and $K R^{*}(K)=r_{\text {off }} z_{0}(K)$. From the above it follows immediately that

$$
\frac{\partial^{2} \hat{\Pi}}{\partial p_{i}^{2}}\left(p^{*}(K), R^{*}(K)\right)<0,
$$

and

$$
\begin{gathered}
\frac{\partial^{2} \hat{\Pi}}{\partial p_{i}^{2}}\left(p^{*}(K), R^{*}(K)\right) \cdot \frac{\partial^{2} \hat{\Pi}}{\partial R_{i}^{2}}\left(p^{*}(K), R^{*}(K)\right)-\left(\frac{\partial^{2} \hat{\Pi}}{\partial p_{i} \partial R_{i}}\left(p^{*}(K), R^{*}(K)\right)\right)^{2}= \\
\frac{(\alpha-1)^{2}}{\alpha} \frac{M}{p^{*}(K)}\left(2 a^{\prime}\left(r_{\mathrm{off}} z_{0}(K)\right)+R_{i} a^{\prime \prime}\left(r_{\mathrm{off}} z_{0}(K)\right)\right)= \\
\frac{(\alpha-1)^{2}}{\alpha} \frac{M}{p^{*}(K)} \frac{f_{m}}{r_{\mathrm{off}}} \frac{1}{\left(1-z_{0}(K)\right)^{3 / 2}}\left(1+\frac{3}{4 K} \frac{z_{0}(K)}{1-z_{0}(K)}\right)>0 .
\end{gathered}
$$

Thus, we have shown that $\left(p^{*}(K), R^{*}(K)\right)$ is a local maximum. Actually, we have proved more, namely that it is the only local maximum over the points of $\mathcal{D}_{i}\left(R^{*}(K) \cdot \mathbf{1}\right)$ at which the second partial derivatives of $\hat{\Pi}_{i}(p, R)$ exist: all but $(0,0)$, i.e., $\mathcal{D}_{i}\left(R^{*}(K) \cdot \mathbf{1}\right) \backslash(0,0)=(0, \infty) \times\left(0, R_{m}^{\prime}\right)$, where $R_{m}^{\prime}=r_{\text {off }}-(K-1) R^{*}(K)$.

We now show that the value of $\hat{\Pi}_{i}$ at the local maximum, $\left(p^{*}(K), R^{*}(K)\right)$, is positive and, in particular, 
greater than the value at $(0,0)$. Indeed,

$$
\begin{aligned}
& \hat{\Pi}_{i}\left(p^{*}(K), R^{*}(K)\right)= \\
& M \frac{r_{\mathrm{off}}}{K} z_{0}(K)\left(\frac{\alpha}{\alpha-1}-K\right)^{1 / \alpha} \frac{1}{\frac{\alpha}{\alpha-1}-K+K}-\frac{r_{\mathrm{off}}}{K} z_{0}(K) a\left(r_{\mathrm{off}} z_{0}(K)\right)= \\
& \frac{r_{\mathrm{off}}}{K} f_{m} z_{0}(K) \sqrt{A(K)}\left(1-\frac{1}{\sqrt{A(K)\left(1-z_{0}(K)\right)}}\right)= \\
& \frac{r_{\mathrm{off}}}{K} f_{m} z_{0}^{2}(K) \sqrt{A(K)} \frac{1-1 / \beta}{1-z_{0}(K) / \beta}>0=\hat{\Pi}_{i}(0,0),
\end{aligned}
$$

where the second equality follows from the fact that

$$
A(K)\left(1-z_{0}(K)\right)^{3}=\left(1-\frac{z_{0}(K)}{\beta}\right)^{2} .
$$

Now, by observing that

$$
\begin{aligned}
\lim _{p \rightarrow 0} \hat{\Pi}_{i}(p, R) & =-R a\left(R+(K-1) R^{*}(K)\right)<0 \\
\lim _{p \rightarrow \infty} \hat{\Pi}_{i}(p, R) & =-R a\left(R+(K-1) R^{*}(K)\right)<0 \\
\lim _{R \rightarrow 0} \hat{\Pi}_{i}(p, R) & =0 \\
\lim _{R \rightarrow R_{m}^{\prime}} \hat{\Pi}_{i}(p, R) & =M p \lim _{R \rightarrow R_{m}^{\prime}} \rho_{i}-R_{m}^{\prime} \lim _{R \rightarrow R_{m}^{\prime}} a(R) \leq M p-R_{m}^{\prime} \lim _{R \rightarrow R_{m}^{\prime}} a(R)=-\infty,
\end{aligned}
$$

we conclude that $\left(p^{*}(K), R^{*}(K)\right)$ is a global maximum in $\mathcal{D}_{i}\left(R^{*}(K) \cdot \mathbf{1}\right)$, which completes the proof.

Figures 3 and 4 show a characterization of the symmetrical equilibrium where all SPs $(K)$ join the market. The existence of this equilibrium is guaranteed, since $K<\eta_{M}$, by Proposition 5 , and the values of prices and data rates in the equilibrium are given in Proposition 4. The results in these figures have been obtained for $M=1000, f_{m}=1$, and several values of $K$. Figure 3 shows the aggregate data rate received by all the providers in the equilibrium as a fraction of the offered rate $\left(K R^{*}(K) / r_{\text {off }}\right)$ and Fig. 4 shows the data-rate-to-price ratio $\left(R^{*}(K) / p^{*}(K)\right)$ in equilibrium for each provider.

Proposition 6. Under the hypotheses of Proposition 4 and with the necessary conditions derived therein, with $\kappa\left(\boldsymbol{p}^{*}, \boldsymbol{R}^{*}\right)<K$ (i.e., some SPs stay outside the market), the strategic profile $\left(\boldsymbol{p}^{*}, \boldsymbol{R}^{*}\right)$ is an equilibrium if, and only if,

$$
\alpha^{1 / \alpha} \geq \sqrt{A(\kappa)\left(1-z_{0}(\kappa)\right)} .
$$

Proof. Let us assume, without any loss of generality, that the SPs are numbered in such a way that

$$
\begin{aligned}
\left(p_{i}^{*}, R_{i}^{*}\right) & =\left(p^{*}(\kappa), R^{*}(\kappa)\right), & & i=1,2, \ldots, \kappa \\
\left(p_{i}^{*}, R_{i}^{*}\right) & =(0,0), & i & =\kappa+1, \kappa+2, \ldots, K .
\end{aligned}
$$

We need to prove that

$$
\Pi_{i}\left(p, R ; \boldsymbol{p}_{-i}^{*}, \boldsymbol{R}_{-i}^{*}\right) \leq \Pi_{i}\left(\boldsymbol{p}^{*}, \boldsymbol{R}^{*}\right) \quad \forall(p, R) \in \mathcal{D}_{i}\left(\boldsymbol{R}_{-i}^{*}\right), i=1, \ldots, \kappa, \kappa+1, \ldots, K .
$$

When $i=1, \ldots, \kappa$ the proof of (48) is exactly analogous to that of Proposition 5 . 




Fig. 3: Aggregate data rate received by the SPs Fig. 4: Data-rate-to-price ratio $\left(R^{*}(K) / p^{*}(K)\right)$ for each SP. $\left(K R^{*}(K) / r_{\text {off }}\right)$.

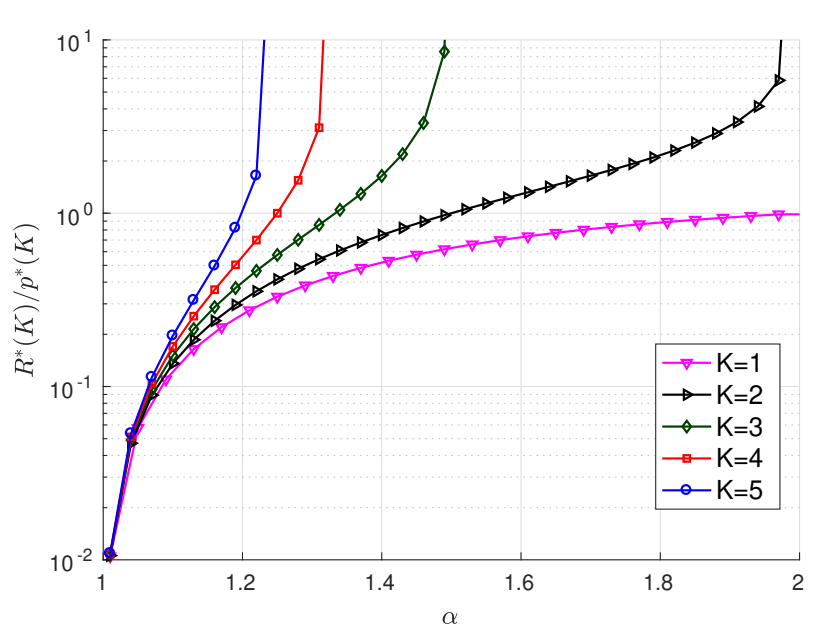

Next we give the proof for the case $i=\kappa+1, \kappa+2, \ldots, K$. In this case (48) can be rewritten as

$$
\begin{aligned}
\Pi_{i}\left(p, R ; \boldsymbol{p}_{-i}^{*}, \boldsymbol{R}_{-i}^{*}\right)=M p \frac{(R / p)^{\alpha}}{1+(R / p)^{\alpha}+\kappa\left(R^{*}(\kappa) / p^{*}(\kappa)\right)^{\alpha}}-a\left(R+\kappa R^{*}(\kappa)\right) & \\
\leq \Pi_{i}\left(\boldsymbol{p}^{*}, \boldsymbol{R}^{*}\right)=0, & \forall(p, R) \in \mathcal{D}_{i}\left(\boldsymbol{R}_{-i}^{*}\right),
\end{aligned}
$$

which can be simplified to

$$
\frac{(R / p)^{\alpha-1}}{(R / p)^{\alpha}+\frac{\alpha}{\alpha-1}\left(\frac{\alpha}{\alpha-1}-\kappa\right)^{-1}} \leq \frac{f_{m} / M}{\sqrt{1-z_{0}(\kappa)-R / r_{\text {off }}}} \quad \forall(p, R) \in \mathcal{D}_{i}\left(\boldsymbol{R}_{-i}^{*}\right) .
$$

The above is equivalent to

$$
\sup _{R / p>0} \frac{(R / p)^{\alpha-1}}{(R / p)^{\alpha}+\frac{\alpha}{\alpha-1}\left(\frac{\alpha}{\alpha-1}-\kappa\right)^{-1}} \leq \inf _{R>0} \frac{f_{m} / M}{\sqrt{1-z_{0}(\kappa)-R / r_{\text {off }}}} .
$$

From this, by application of Lemmas 2 and 1, we obtain the following equivalent condition

$$
\frac{f_{m}}{M} \frac{\sqrt{A(\kappa)}}{\alpha^{1 / \alpha}} \leq \frac{f_{m} / M}{\sqrt{1-z_{0}(\kappa)}}
$$

which completes the proof.

Figure 5 illustrates the condition (47) (given in Proposition 6) for $M=1000, f_{m}=1$ and several values of $k$. We have plotted the left-hand side of the inequality $\left(\alpha^{1 / \alpha}\right)$, and the right-hand side $\left(\sqrt{A(k)\left(1-z_{0}(k)\right)}\right)$ for $k=4,5,6,7$. For a given value of $k<K$, the equilibrium exists if, and only if, the curve of $\sqrt{A(k)\left(1-z_{0}(k)\right)}$ is below the curve of $\alpha^{1 / \alpha}$ and the condition $k<\eta_{M}$ is fulfilled.

Using the definition of $z_{0}(k)$ we can write

$$
\sqrt{A(k)\left(1-z_{0}(k)\right)}=\frac{1-z_{0}(k) / \beta}{1-z_{0}(k)}>1
$$




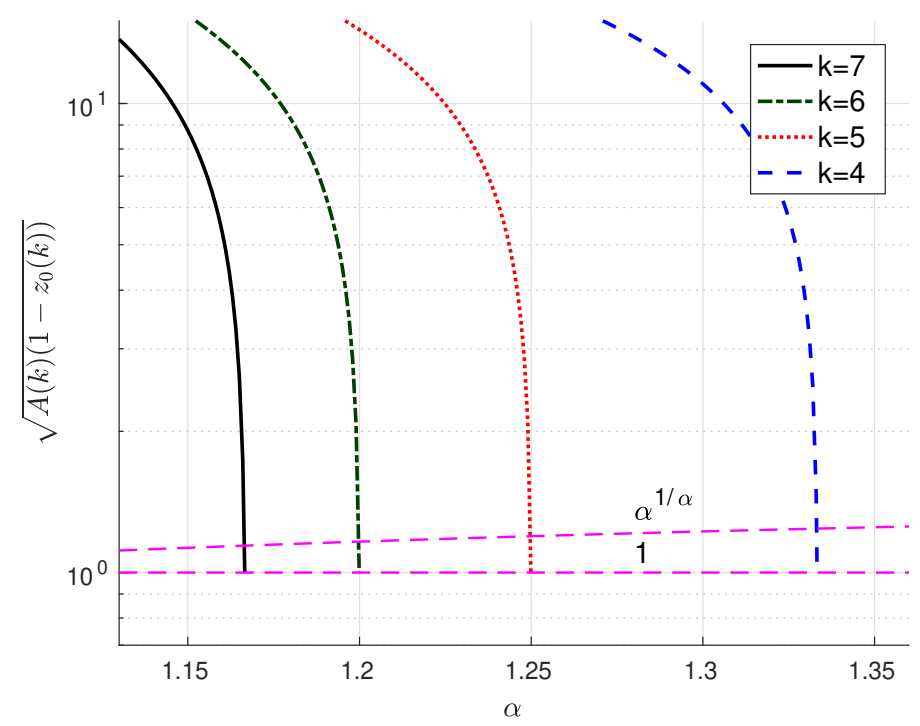

Fig. 5: Condition for the existence of an equilibrium with $k$ SPs (out of $K$ ) entering the market.

the last inequality being a consequence of the fact that $z_{0}(k)>0$. Besides, it easy to check that when $\alpha$ approaches the value at which $\eta_{M}=k, A(k)$ approaches $1, z_{0}(k)$ approaches 0 and, consequently, $\sqrt{A(k)\left(1-z_{0}(k)\right)}$ approaches 1 . Therefore, for each $k<K$, the equilibrium only exists if value of $\alpha$ is in the extremely narrow interval in which the curve $\sqrt{A(k)\left(1-z_{0}(k)\right)}$ is between $\alpha^{1 / \alpha}$ and 1 . To further illustrate this, let $\alpha_{m}(k)$ and $\alpha_{M}(k)$ be, respectively, the lower and upper limits of the interval, that is,

$$
\begin{aligned}
\sqrt{A(k)\left(1-z_{0}(k)\right)} & =\alpha^{1 / \alpha}, \quad \text { if } \alpha=\alpha_{m}(k) \\
\lim _{\alpha \rightarrow \alpha_{M}(k)} \sqrt{A(k)\left(1-z_{0}(k)\right)} & =1 .
\end{aligned}
$$

Thus, for a given $k<K$, if $\alpha \in\left[\alpha_{m}(k), \alpha_{M}(k)\right)$ the two conditions hold and there exist equilibria in which $k$ out of $K$ SPs join the market.

Figure 6 shows the upper limit of the interval, $\alpha_{M}(k)$, as a function of $k$ for different values of $f_{m} / M$. Similarly, the length of the interval, $\alpha_{M}(k)-\alpha_{M}(k)$, is shown in Fig. 7. The results in Fig. 7 confirm that this type of equilibrium can only exist for a very narrow range of the sensitivity parameter $\alpha$. As shown in Fig. 6 the position of the interval is rather insensitive to the value of $f_{m} / M$ and it approaches 1 as $k$ grows. The observation of both graphs also reveals that, for a given value of $f_{m} / M$, there is no overlap between the intervals corresponding to different values of $k$. This means that in those infrequent cases where the value of the sensitivity is such that there is an equilibrium with only a part of the SPs joining the market, the number of SPs that join the market takes a definite and unique value: $k=\left\lfloor\eta_{M}(\alpha)\right\rfloor<\eta_{M}(\alpha) \leq K$.

\subsection{Summary}

If users' sensitivity to the data-rate-to-price ratio is not above the unity $(\alpha \leq 1)$ there is no Nash equilibrium $(\alpha<1)$ or the only possible equilibrium is one with no practical interest $(\alpha=1)$, where there would really not be any business since no SPs participate in the market.

When the users' sensitivity is above the unity and the number of competing SPs is less that $\eta_{M}$, there exists a unique Nash equilibrium. In this equilibrium all SPs join the market and utilize the same strategy. If the number of SPs is not below $\eta_{M}$, there might exist some equilibria where only a subset of $k$ SPs (with $\left.1 \leq k=\left\lfloor\eta_{M}\right\rfloor<\eta_{M} \leq K\right)$ join the market and utilize the same strategy, but this is only possible for a very narrow range values of the sensitivity parameter $\alpha$. 


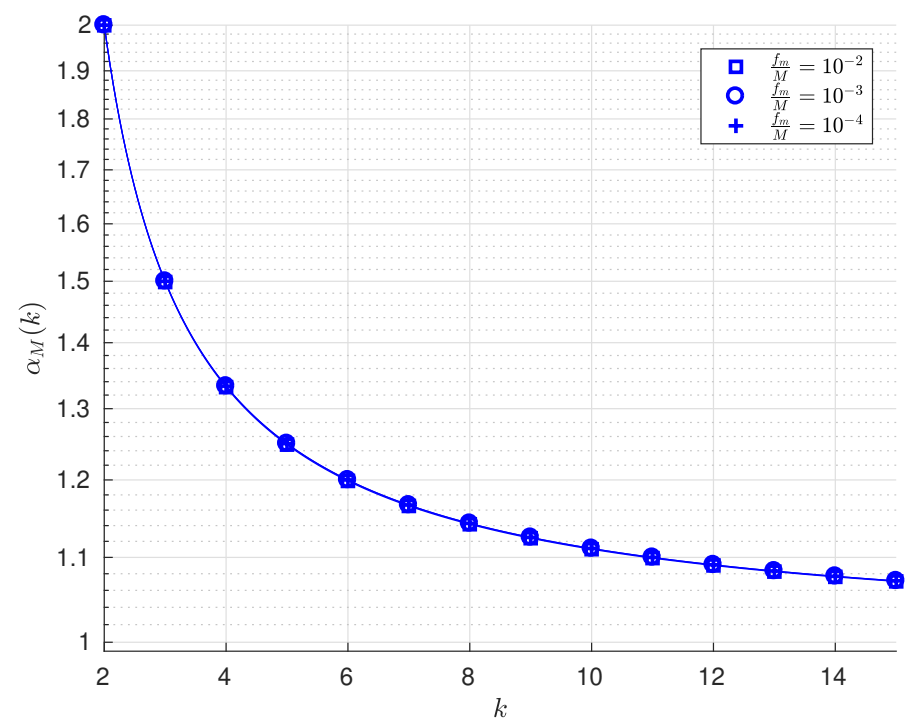

Fig. 6: Upper limit of the interval of $\alpha$ where equilibria with $k<K$ SPs join the market.

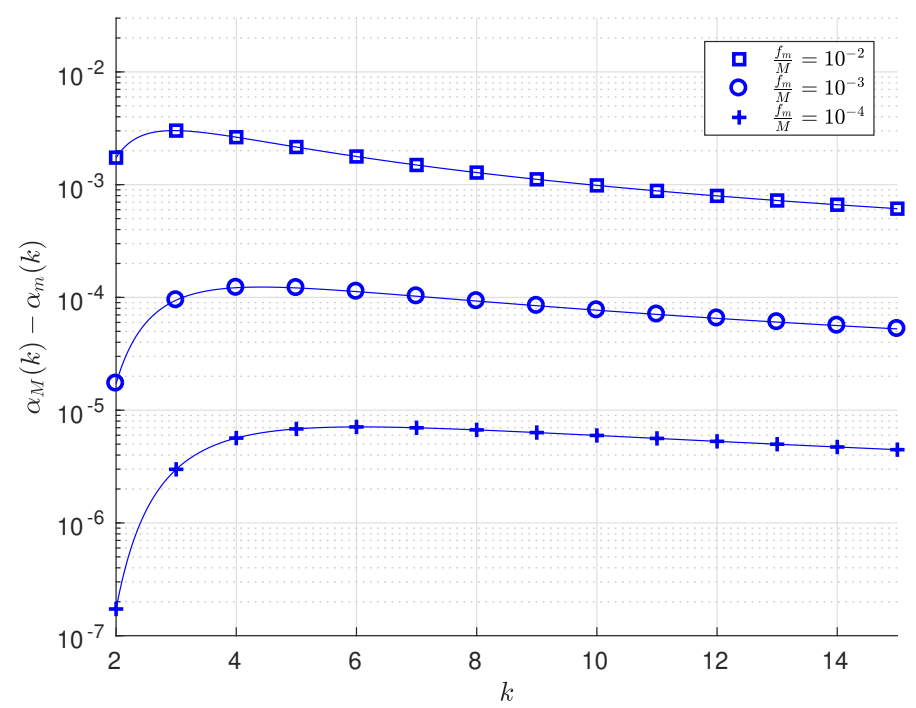

Fig. 7: Length of the interval of $\alpha$ where equilibria with $k<K$ SPs join the market. 


\section{Results and discussion}

In this section, we explore the characteristics of the competition game Nash equilibria when the equilibrium is different from $(\mathbf{0}, \mathbf{0})$. First, we analyze the effect of the size of the market, represented by the number of SPs (or, equivalently, by the indicator of competition level) and the number of users. Next, we explore the strategic decision taken by SPs, concerning the data rate and the price. Finally, we discuss the profits of the stakeholders in symmetric equilibrium.

All the results in this section have been obtained considering the following parameter values: $f_{m}=1 \mathrm{cu}$, $r_{\max }=1 \mathrm{nbps}, R_{0}=1 \mathrm{nbps}$, and $p_{0}=1 \mathrm{cu}$.

\subsection{Intensity of competition}

In order to describe the resulting intensity of competition, we adopt the most widespread indicator: the Hirschman-Herfindahl Index, or HHI for short (see [28] for its definition and [29] for its sensitivity analysis that claims its superiority over other indices). The HHI is a numerical index taking values in the interval $(0,1]$, with large values corresponding to a low intensity of competition (i.e., close to a monopoly, which is represented by the value $\mathrm{HHI}=1$ ), and vice versa. In the special case of symmetry among SPs, the HHI boils down to the inverse of the number of SPs joining the market $(1 / \kappa)$.

Let us first see what the condition is so that at least one SP joins the market. If we impose the condition $\eta_{M}>1$ in the expression of the upper bound on the number of SPs given by (37), we obtain

$$
\frac{f_{m}}{M}<\frac{(\alpha-1)^{\frac{\alpha-1}{\alpha}}}{\alpha} .
$$

When $\alpha$ varies in $[1, \infty)$ the minimum value of the right hand side of $(52)$ is 0.5 (see Lemma 3 ). Thus, if $f_{m} / M<0.5$ the condition is satisfied for all values of $\alpha \geq 1$.

With regard to the intensity of competition, the maximum number of SPs that join the market in equilibrium is reached when $K=\left\lfloor\eta_{M}\right\rfloor$, in which case $\kappa=K$, and

$$
\mathrm{HHI}=\frac{1}{\left\lfloor\frac{\alpha}{\alpha-1}-\left(\frac{\alpha}{\alpha-1} \frac{f_{m}}{M}\right)^{\alpha}\right\rfloor} .
$$

We see that the maximum intensity of competition depends on both $\alpha$ and $f_{m} / M$, but their importance is far from being equal. In Fig. 8, we see that the intensity of competition is driven mainly by the users' sensitivity $\alpha$, with a very small impact of $f_{m} / M$. In a large interval (roughly for $1.5<\alpha<2$ ), we have a duopoly (HHI $=0.5)$, while a significant competition arises when $\alpha<1.3$. This can be interpreted as follows. The higher the sensitivity $\alpha$, the more difficult the competition between the SPs is. As a consequence, for high values of $\alpha$, only equilibria with a reduced number of competitors are possible, while for values of $\alpha$ slightly above 1 , the number of SPs that can compete in equilibrium increases.

\subsection{Penetration ratios}

It may be expected that different intensities of competition among SPs have an impact on the number of subscribers.

When an SP operates as a monopoly, the penetration ratio, defined as the ratio between the number of subscribers and the number of users, is generally low, and it approaches zero when $\alpha$ approaches 1 [30]. When there is competition between more than one SP, the total penetration ratio is instead equal to $K$ times the penetration ratio of any single SP. From (40), and assuming $K=\left\lfloor\eta_{M}\right\rfloor$,

$$
\rho_{\mathrm{tot}}=K\left(1-\frac{1}{\alpha}\right)=\left\lfloor\frac{\alpha}{\alpha-1}-\left(\frac{\alpha}{\alpha-1} \frac{f_{m}}{M}\right)^{\alpha}\right\rfloor\left(1-\frac{1}{\alpha}\right) .
$$

In Fig. 9, where $M=1000$, we show that the limitation on the penetration ratio can be transcended by allowing competition: the penetration ratio is always higher in competition than in a monopoly, and the difference increases as $\alpha$ approaches 1 . 


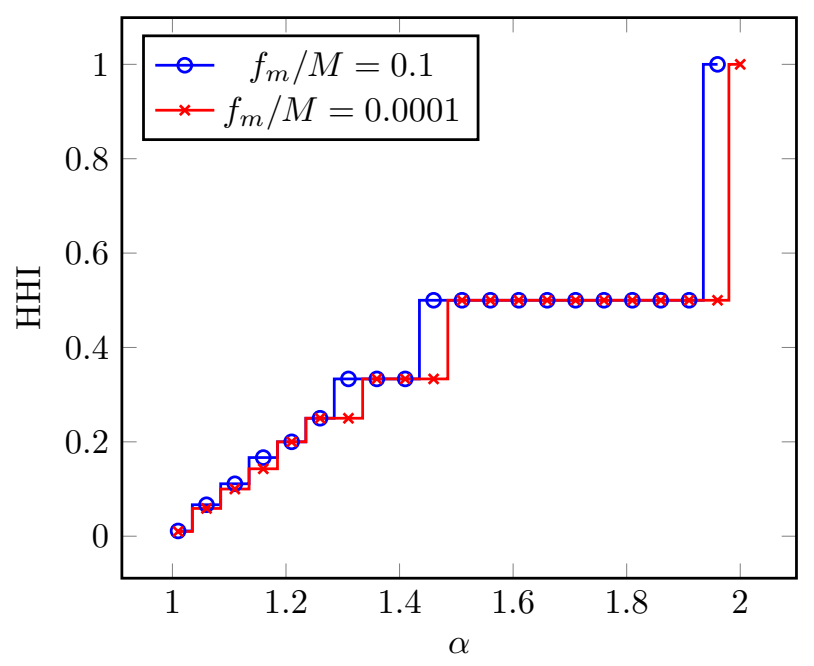

Fig. 8: Hirschman-Herfindahl Index

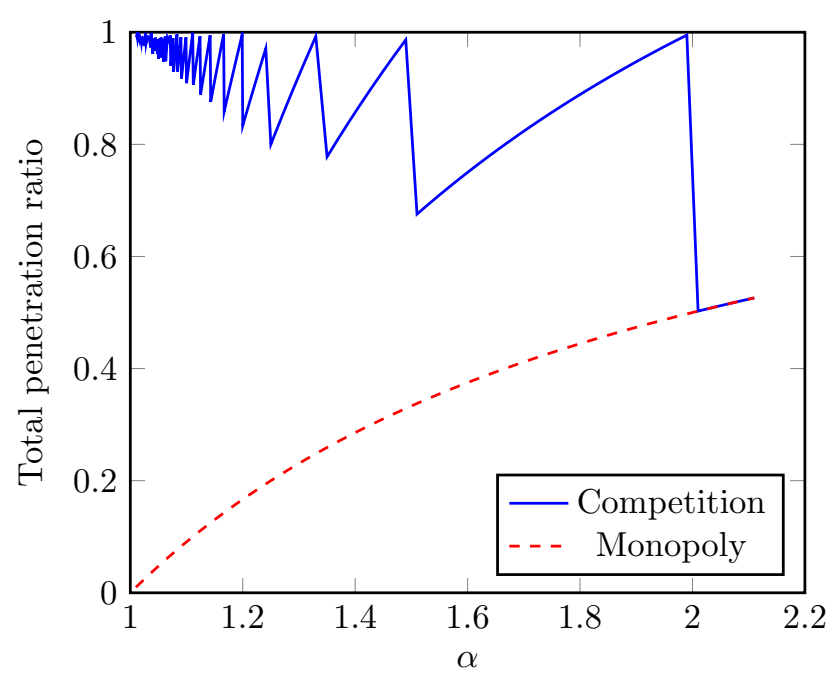

Fig. 9: Service Penetration Ratio 


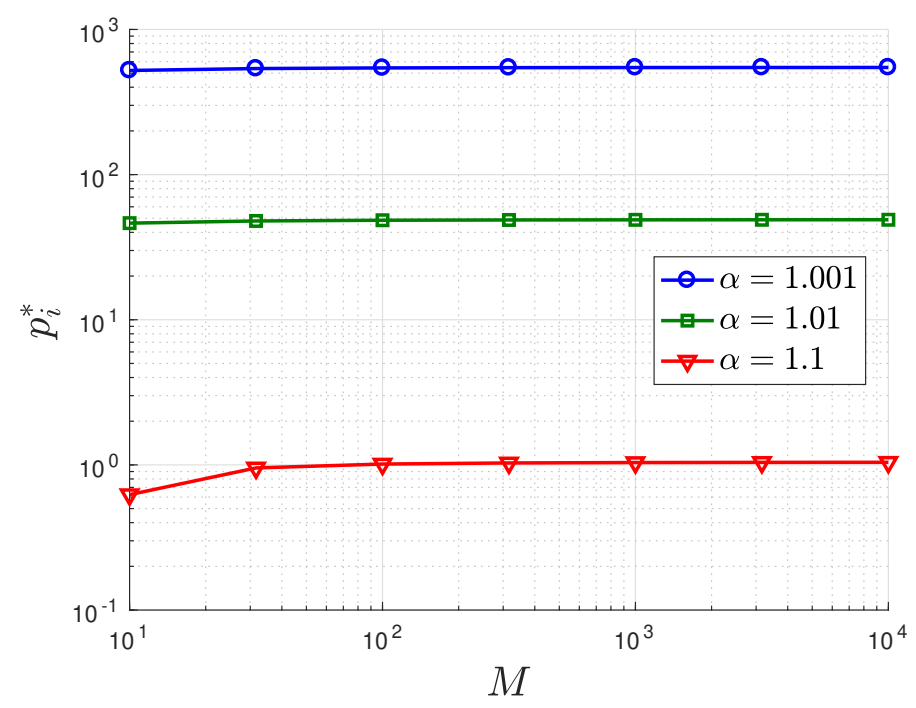

Fig. 10: $p_{i}$ in equilibrium as a function of $M$ for different $\alpha$ values

\subsection{Data rate and price}

Each SP's strategy consists of a price $p_{i}$ and a data rate $R_{i}$. In this section we discuss the results obtained in the symmetric equilibrium for both values and for the ratio $p_{i}^{*} / R_{i}^{*}$, which is the price that each user pays for each unit of processed data. These values are given by (39), (38) and the inverse of (43). All results presented in this subsection have been obtained for a number of DPs $N=10$.

The three parameters that define the market size are the number $M$ of users, the number $N$ of DPs, and the number $K$ of SPs, and each one impacts differently on the price.

The impact of the number $M$ of users on the price is negligible as seen in Fig. 10, where $K=9$ and $\alpha=1.1,1.01,1.001$, and an equilibrium exists for $k=K$, i.e., it can be checked that for these values of $\alpha$ and of $K$ an equilibrium for $k=K$ exists. The impact of $M$ on $R_{i}^{*}$ is also negligible, as seen in Fig. 11, except for very low values of $M$. Therefore, the influence of $M$ on $\left(R_{i}^{*}, p_{i}^{*}\right)$ is negligible as long as $M$ is above a critical amount. In the case discussed here this critical amount is less than $M=100$. Note that values lower than 100 are unrealistic.

Regarding the influence of the number $N$ of DPs on the price and on the data rate, both values grow proportionally with $N$. In fact, from (38) and (5), $R_{i}^{*}$ is proportional to $N$ because $z_{0}(k)$ does not depend on $N$, and from (39), $p_{i}^{*}$ is proportional to $R_{i}^{*}$. As a consequence, $p_{i}^{*} / R_{i}^{*}$ does not depend on $N$.

In Figs. 12, 13 and 14, we can see that $p_{i}^{*}, R_{i}^{*}$ and $p_{i}^{*} / R_{i}^{*}$ decrease non linearly with the the number $K$ of SPs. Now, $M=1000$ and again it can be checked that an equilibrium for $k=K$ exists for all $\alpha$ and $K$ plotted in the curves.

As expected, Fig. 12 shows that an increased intensity of competition results in a falling price $p_{i}^{*}$. The effect is similar for the different values of $\alpha$. Nevertheless, $\alpha$ itself has a strong influence on the price, since, for a given $K$, a slight increase of $\alpha$ results in a strong decrease in $p_{i}^{*}$.

The data rate also decreases with $K$ (Fig. 13) because the data supplied by the DPs is not reused among the SPs, but instead it must be split among the $K$ SPs. Note that $R_{i}^{*}$ is very close to $r_{\text {off }} / K$-for the current parameters values, $r_{\text {off }}=5$.

Finally, in Fig. 14, we see that the influence of $K$ on $p_{i}^{*} / R_{i}^{*}$ becomes sharper as the number of competitors approaches the maximum allowed by $\alpha$. When the number of competitors is low, as when $\alpha=1.001$ and $\alpha=1.01, p_{i}^{*} / R_{i}^{*}$ decreases almost negligibly — in these cases the maximum value of $K$ is much greater than 9 . When the number of competitors is high, as when $\alpha=1.1, p_{i}^{*} / R_{i}^{*}$ drops clearly as the number of competitors approaches 9 - which is the maximum value of $K$. 




Fig. 11: $R_{i}$ in equilibrium as a function of $M$ for different $\alpha$ values

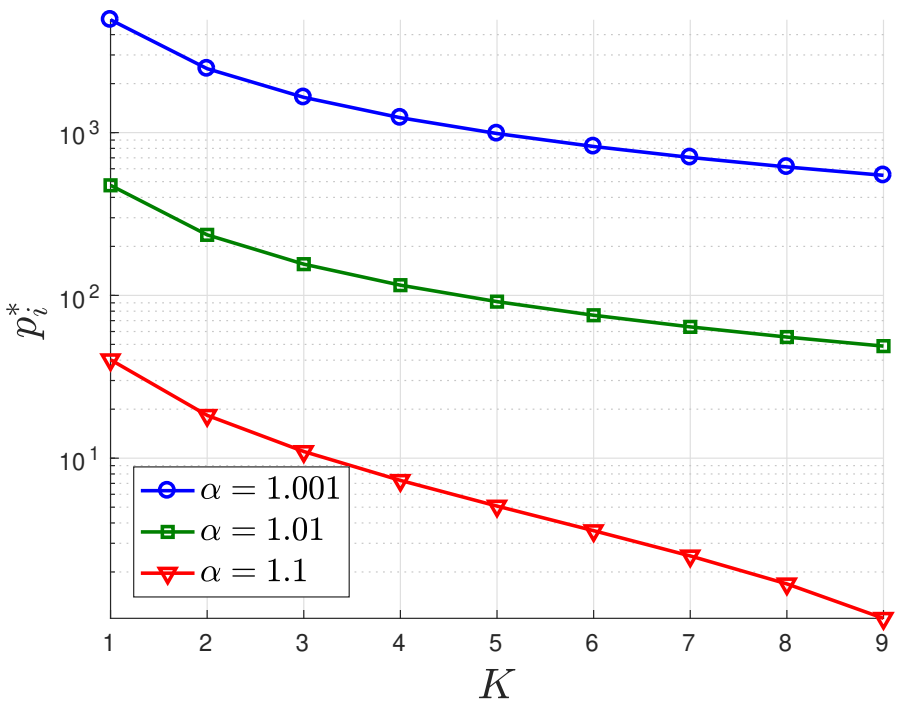

Fig. 12: $p_{i}$ in equilibrium as a function of $K$ for different $\alpha$ values 


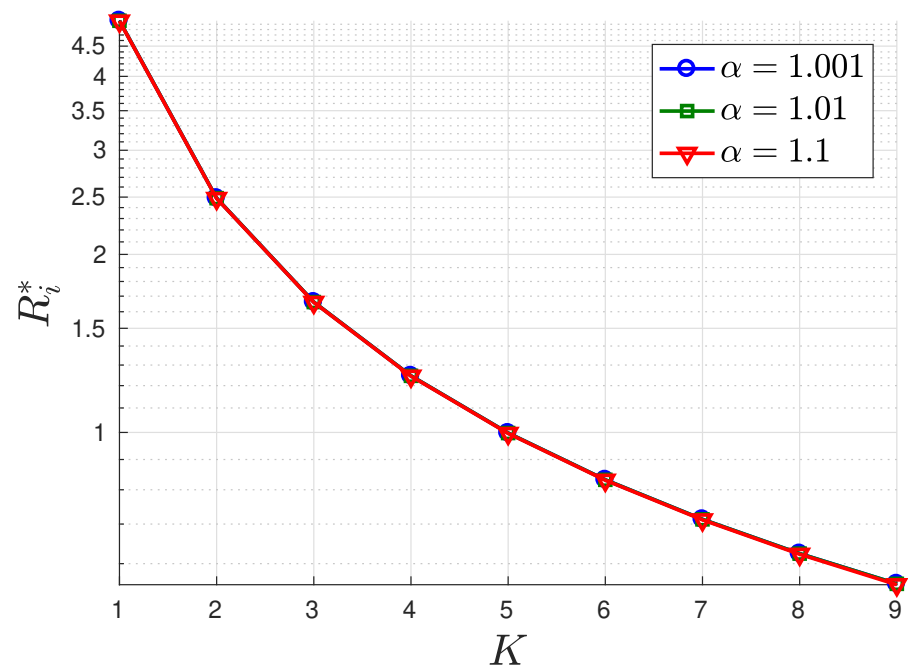

Fig. 13: $R_{i}$ in equilibrium as a function of $K$ for different $\alpha$ values

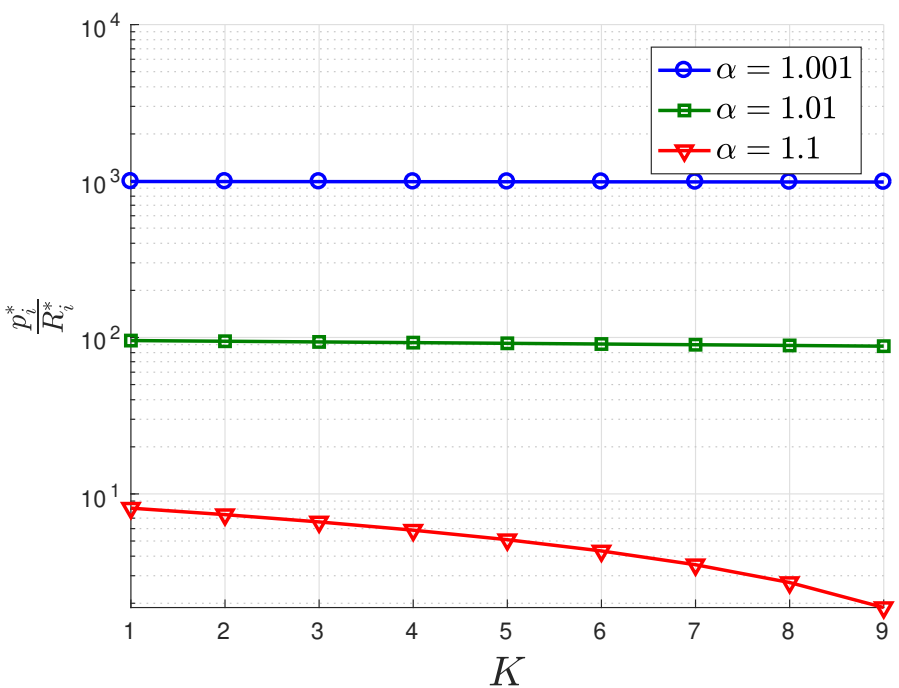

Fig. 14: $p_{i} / R_{i}$ in equilibrium as a function of $K$ for different $\alpha$ values 


\subsection{Profits}

Next we examine the surplus of each agent in the equilibrium. All results presented in this subsection have been obtained for $M=1000$ users, $N=10$ DPs and $K=9$ SPs.

Fig. 15 shows the aggregate profit of all SPs, $\Pi_{S P s}$, given by (13), as a function of $K$ for three values of $\alpha$ for which an equilibrium for $k=K$ exists. We see that the profit decreases as the number of competitors $K$ increases. This is because, as explained in the previous section, a more intense competition translates into lower prices for users, while the total amount of data acquired by the SPs $\left(K R_{i}^{*}\right)$ remains practically constant. Again we see that the influence of $K$ on the aggregate profit becomes sharper when $\alpha=1.1$, since here $K$ approaches the maximum number of competitors allowed.

This result can be generalized for any number of DPs $N$, since from (13), (38), (39), (40) and (6),

$$
\Pi_{\mathrm{SPs}}=\left[M\left(\frac{\alpha}{\alpha-1}-k\right)^{1 / \alpha}\left(1-\frac{1}{\alpha}\right)-\frac{f_{m}}{\sqrt{1-z_{0}(k)}}\right] \frac{r_{\max } z_{0}(k)}{2} N,
$$

so $\Pi_{S P s}$ is proportional to $N$. Therefore the curves of $\Pi_{S P s}$ for values of $N \neq 10$ would have the same shape as those of Fig. 15, but with a vertical offset.

Fig. 16 shows the aggregate surplus of all users as a function of $K$ for three values of $\alpha$. We see that the users' surplus increases as the number of competitors $K$ increases, bearing in mind that, as discussed above, $p_{i}^{*} / R_{i}^{*}$ decreases with competition. The variation is again sharper when $K$ is closer to the maximum allowed by $\alpha$ (see the curve for $\alpha=1.1$ ). Besides, the influence of $\alpha$ on the price translates into a strong influence in the users' surplus, so that more sensitive users will be able to extract more surplus from the service.

In Fig. 17, the DPs' surplus $\Pi_{D P s}$, given by (10), is represented as a function of $K$ for the three values of $\alpha$. In this case, the effect of competition is not straightforward. The overall effect can be observed in the shape of the curve for $\alpha=1.1$, since for this $\alpha$ value the plot contains all the $K$ values for which an equilibrium exists $(1 \leq K \leq 9)$. We can see that, for a low intensity of competition, the DPs' surplus increases with the competition, while for a high intensity, it decreases with $K$, reaching a maximum at about half the range of possible $K$ values. In the same way as with the SPs' surplus, the DPs' surplus is seriously affected by the value of the sensitivity $\alpha$. As $\alpha$ increases, $\Pi_{D P s}$ decreases, since a more intense competition translates into a lower unit price $a$ paid at the DPs' side. Additionally, $\Pi_{D P s}$ increases as $N$ increases, since the offered rate $r_{\text {off }}$ increases and also the total rate requested by the SPs. Like $\Pi_{S P s}$, DPs' surplus increases proportionally to $N$, since, from (6) and (10), the expression of DPs' surplus can be written as

$$
\Pi_{\mathrm{DPs}}=\frac{f_{m}}{\sqrt{1-z_{0}(k)}}\left(1-\sqrt{1-z_{0}(k)}\right)^{2} \frac{r_{\max }}{2} N .
$$

Taking the overall effect of $\alpha$, we can conclude that a high users' sensitivity to the data-rate-to-price ratio causes a surplus shift from both the SPs and DPs to the users.

\section{Conclusions}

We have applied game theory to analyze the business model of a scenario made up of data providers, service providers and users. A thorough mathematical analysis of the Nash equilibria is carried out and the following results have been proved:

- Equilibria can only exist when the users' sensitivity to the data-rate-to-price ratio is greater than the unity.

- When equilibria exist, two mutually exclusive equilibrium types are possible: a trivial equilibrium in which no service provider enters the market, and a symmetric equilibrium in which a number of service providers enter the market and choose the same strategy (data rate and price).

- The non-trivial symmetric equilibrium type is only possible if the number of users is large enough. 


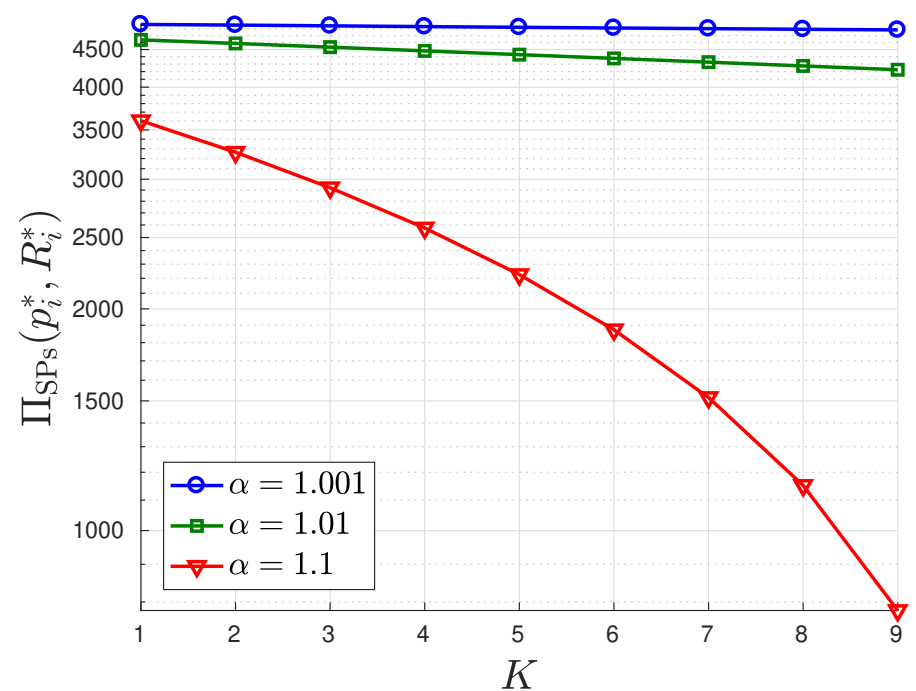

Fig. 15: $\Pi_{S P s}$ in equilibrium as a function of $K$ for different $\alpha$ values.

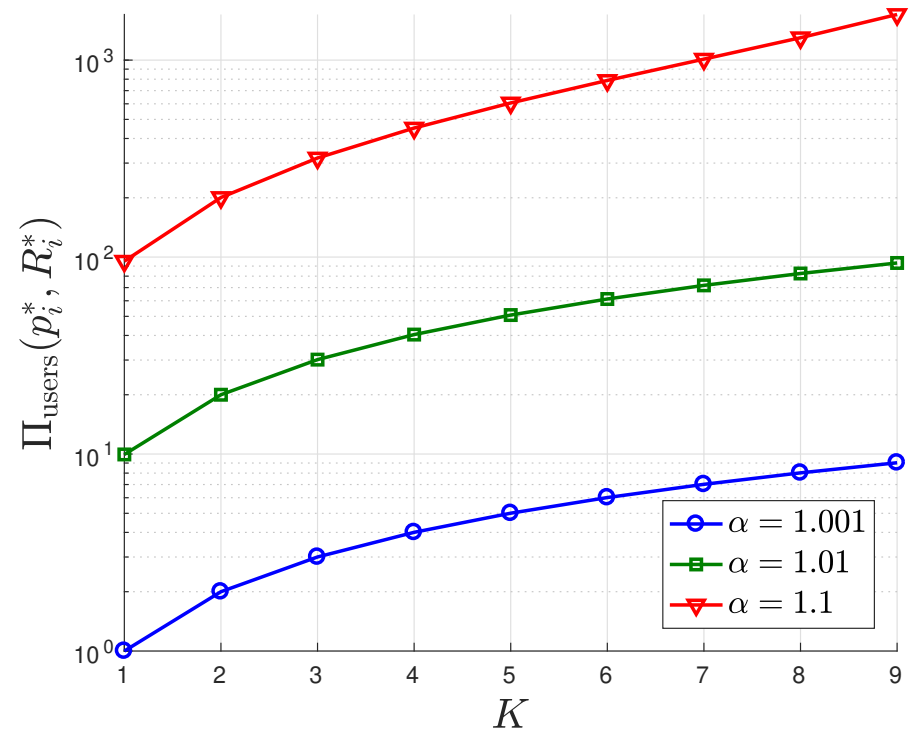

Fig. 16: Mean user surplus in equilibrium as a function of $K$ for different $\alpha$ values. 


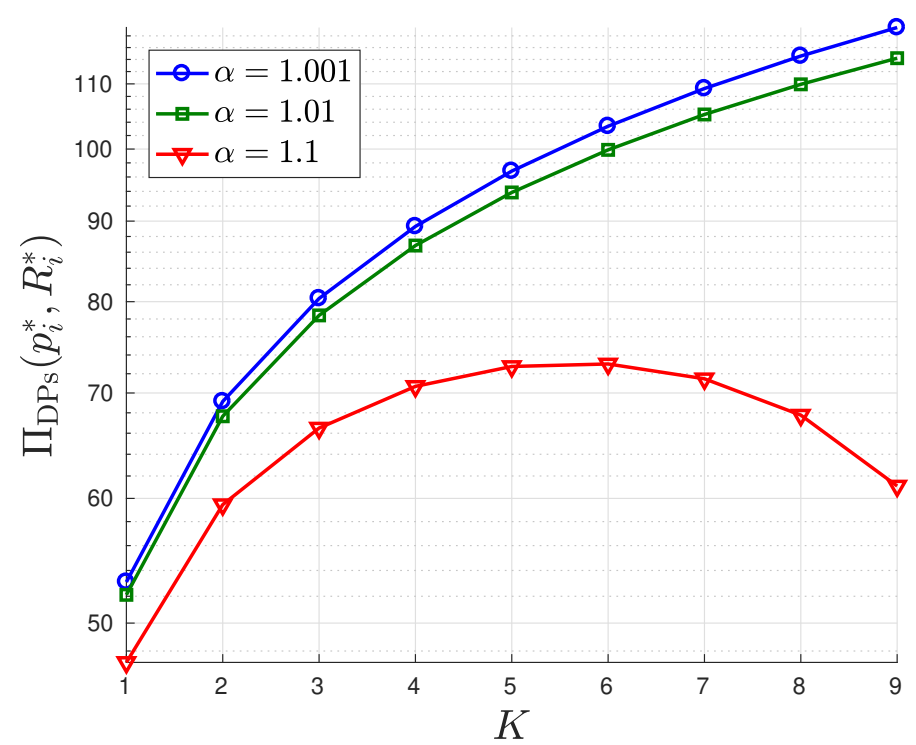

Fig. 17: DPs' surplus in equilibrium as a function of $K$ for different $\alpha$ values.

- In a symmetric equilibrium, the number of active service providers is limited by a value that depends on the number of users and on the sensitivity. If the number of service providers is not greater than this bound, the most feasible equilibrium is the one in which all the service providers enter the market.

- Symmetric equilibria where not all the active service providers enter the market may also exist, although this is only possible under very specific conditions that are very unlikely in most real settings.

- The intensity of competition is beneficial for the users and detrimental for the service providers, as expected. For the data providers, the maximum surplus is obtained at an intermediate intensity of competition.

- An increase in the users' sensitivity to the data-rate-to-price ratio causes a shift on the surplus from both the service providers and data providers to the users. 


\section{Appendix A. Notation}

Introduced in

Equation page

\section{DPs}

Number of DPs

Number of connected DPs (random variable)

Information data generated by DP $j$

Price per unit of data rate charged by DPs

Network access fee payed by each DP to a network access provider

Profit of DP $j$

Expected number of connected DPs

Maximum data rate delivered by a data provider

Average aggregate data rate offered by data providers

Expected aggregate data rate provided by all connected DPs

Inverse supply function

DPs surplus

Service providers (SPs)

Number of SPs

aggregate data rate that SP $i$ obtains from the DPs

Priced charged by SP $i$ to each of its subscribers

Profit of SP $i$

Aggregate profit of all SPs in the symmetric case

\section{Users}

Number of users

Objective part of the utility that a user obtains from SP $i$

Sensitivity parameter

Subjective part of the utility that user $u$ obtains from SP $i$

Users' sensitivity parameter

Number of subscribers to the $i^{\text {th }}$ service provider

Penetration ratio of the $i^{\text {th }}$ service provider among users

Users' surplus

Users' surplus in the symmetric case

\section{Analysis, results and auxiliary notation}

Vector of zeros (ones) of the appropriate dimension

CDF of the continuous uniform distribution in $[0,1]$

Upper bound of $k$ that appears in certain conditions

Nash equilibrium point

Indices of the SPs entering the market

Number of SPs entering the market

Unique solution in $(0,1)$ to $A(k)(1-x)^{3}-\left(1-\frac{x}{\beta}\right)^{2}=0$

$$
=0
$$

$N$

$\mathcal{N} \quad 5$

$r_{j}$

$a$

$f$

$\Pi_{j} \quad(1) \quad 5$

$r_{\max }$

3

$R_{i}$

$p_{i}$

$\Pi_{i}$

$\Pi_{\mathrm{SPs}}^{(s)}$

(9) 7

$\begin{array}{lcl}M & & 3 \\ v_{i} & (7) & 6 \\ \mu & & 6 \\ \kappa_{u, i} & & 6 \\ \alpha & & 6 \\ m_{i} & (8) & 6 \\ \rho_{i} & (11) & 7 \\ \Pi_{\text {users }} & (12) & 7 \\ \Pi_{\text {users }}^{(s)} & \end{array}$

$\mathbf{0} /(\mathbf{1})$

$\Psi(\cdot)$

$\eta_{M}$

$\boldsymbol{p}_{-i}, \boldsymbol{R}_{-i}$

$\mathcal{D}_{i}\left(\boldsymbol{R}_{-i}\right)$

$\left(\boldsymbol{p}^{*}, \boldsymbol{R}^{*}\right)$

$\mathcal{I}(\boldsymbol{p}, \boldsymbol{R})$

$k \equiv k(\boldsymbol{p}, \boldsymbol{R})$

$A(k)$

$\beta$

$z_{0}(k)$

5

(37) $\quad 12$

6

6

8

\begin{tabular}{l}
3 \\
6 \\
6 \\
6 \\
6 \\
6 \\
6 \\
7 \\
\hline
\end{tabular}

9
5
2
5
9
9
1
1
2
2
2

\section{Appendix B. Lemmas}

Lemma 1. Let $B>0$, then

$$
\inf _{x>0} \frac{1}{\sqrt{B-x}}=\frac{1}{\sqrt{B}} .
$$

Proof. The proof is immediate by noting that $f(x)=1 / \sqrt{B-x}$ is increasing in $(0, \infty)$. 
Lemma 2. Let $\alpha>1$ and $B>0$, then

$$
\max _{x>0} \frac{x^{\alpha-1}}{x^{\alpha}+B}=\frac{1}{\alpha}(\alpha-1)^{1-1 / \alpha} B^{-1 / \alpha} .
$$

Proof. Let

$$
f(x)=\frac{x^{\alpha-1}}{x^{\alpha}+B},
$$

which is continuous and differentiable for $x>0$.

Clearly, $f(0)=0, \lim _{x \rightarrow \infty} f(x)=0$ and

$$
f^{\prime}(x)=\frac{x^{\alpha-2}}{\left(x^{\alpha}+B\right)^{2}}\left((\alpha-1) B-x^{\alpha}\right) .
$$

It is easily seen that $f^{\prime}(x)>0$ if $x<x_{0}=((\alpha-1) B)^{1 / \alpha}$ and $f^{\prime}(x)<0$ if $x>x_{0}$.

Therefore,

$$
\max _{x>0} f(x)=f\left(x_{0}\right)=\frac{1}{\alpha}(\alpha-1)^{1-1 / \alpha} B^{-1 / \alpha} .
$$

Lemma 3. Let $f:(1, \infty) \rightarrow \mathbb{R}^{+}$be the function defined by

$$
f(x)=\frac{(x-1)^{1-\frac{1}{x}}}{x}
$$

then

$$
\begin{aligned}
\lim _{x \rightarrow 1^{+}} f(x) & =\lim _{x \rightarrow \infty} f(x)=1 \\
f^{\prime}(x) & =\frac{f(x)}{x^{2}} \log (x-1) \\
f(x) & \text { is decreasing in }(1,2) \text { and increasing in }(2, \infty) \\
\operatorname{Im} f & =\left[\frac{1}{2}, 1\right) .
\end{aligned}
$$

Proof. The proof (B.7) and (B.8) is straightforward, and the rest follows immediately from these two.

Lemma 4. Let $A>0, \beta>1$ and

$$
P(x)=A(1-x)^{3}-\left(1-\frac{x}{\beta}\right)^{2}
$$

Then,

- if $A \leq 1, P(x)$ has no zeros in $(0,1)$,

- if $A>1, P(x)$ has exactly one zero in $(0,1)$.

Proof. If $0<A \leq 1$ and $x \in(0,1)$, then $0<1-x<1-x / \beta<1$, and from this

$$
(1-x)^{2}<\left(1-\frac{x}{\beta}\right)^{2} .
$$

Also,

$$
0<A(1-x)<1 .
$$


Multiplying (B.10) and (B.11) we obtain

$$
A(1-x)^{3}<\left(1-\frac{x}{\beta}\right)^{2},
$$

and from this we have $P(x)<0$.

We now turn to the case $A>1$.

Since $P(0)=A-1>0$ and $P(1)=-(1-1 / \beta)^{2}<0$ we conclude that $P(x)$ has at least one zero in $(0,1)$.

Let $z_{1}^{\prime}, z_{2}^{\prime}\left(z_{1}^{\prime}<z_{2}^{\prime}\right)$ denote the two zeros of

$$
P^{\prime}(x)=\frac{2}{\beta}\left(1-\frac{x}{\beta}\right)-3 A(1-x)^{2} .
$$

We observe that

$$
\begin{aligned}
& P^{\prime}(0)=\frac{2}{\beta}-3 A<2-3<0, \\
& P^{\prime}(1)=\frac{2}{\beta}\left(1-\frac{1}{\beta}\right)>0, \\
& P^{\prime}(\beta)=-3 A(1-\beta)^{2}<0 .
\end{aligned}
$$

Consequently,

$$
0<z_{1}^{\prime}<1<z_{2}^{\prime}<\beta \text {. }
$$

Summarizing, we have

- $P(0)>0$.

- $P^{\prime}(x) \leq 0$, if $x \in\left[0, z_{1}^{\prime}\right]$.

- $P^{\prime}(x) \geq 0$ if $x \in\left[z_{1}^{\prime}, 1\right]$.

- $P(1)<0$.

Combining the inequalities above we deduce that $P(x)$ has a unique zero in $\left(0, z_{1}^{\prime}\right)$, and that $P(x)<0$ if $x \in\left[z_{1}^{\prime}, 1\right)$.

Note that this result is slightly stronger than the second statement of the lemma, since we have proved that the unique zero in $(0,1)$ is actually in $\left(0, z_{1}^{\prime}\right) \subset(0,1)$.

\section{Declarations of interest, funding sources and author contributions}

Declarations of interest: none.

This work has been supported by the Spanish Ministry of Economy and Competitiveness through project TIN2013-47272-C2-1-R.

L. Guijarro proposed the business model and the game model. V. Pla performed the analysis. J.R. Vidal and M. Naldi produced the numerical results. All authors collaboratively wrote the paper. All authors approve the final manuscript.

\section{References}

[1] IDC, European Data Market SMART 2013/0063. Final Report, Tech. rep. (2017).

URL http: //datalandscape.eu/study-reports/european-data-market-study-final-report

[2] Accenture, Value of data. the dawn of the data marketplace, Tech. rep. (2018).

URL https://www.accenture.com/us-en/insights/high-tech/dawn-of-data-marketplace 
[3] J. Deichmann, K. Heineke, T. Reinbacher, D. Wee, Creating a successful internet of things data marketplace. URL http://www.mckinsey.com/business-functions/digital-mckinsey/our-insights/creating-a-successfulinternet-of-things-data-marketplace

[4] F. Stahl, F. Schomm, G. Vossen, L. Vomfell, A classification framework for data marketplaces, Vietnam Journal of Computer Science 3 (3) (2016) 137-143.

[5] EuropeanCommission, Towards a common european data space (COM(2018) 232).

[6] J. Von Neumann, O. Morgenstern, Theory of games and economic behavior, Princeton university press, 1953.

[7] X. Vives, Oligopoly pricing: old ideas and new tools, MIT press, 2001.

[8] M. Cagalj, S. Ganeriwal, I. Aad, J.-P. Hubaux, On selfish behavior in csma/ca networks, in: INFOCOM 2005. 24th Annual Joint Conference of the IEEE Computer and Communications Societies. Proceedings IEEE, Vol. 4, IEEE, 2005, pp. 2513-2524.

[9] R. Johari, J. N. Tsitsiklis, Efficiency loss in a network resource allocation game, Mathematics of Operations Research 29 (3) (2004) 407-435.

[10] J.-J. Laffont, S. Marcus, P. Rey, J. Tirole, Internet interconnection and the off-net-cost pricing principle, RAND Journal of Economics (2003) 370-390.

[11] S. Shakkottai, R. Srikant, Economics of network pricing with multiple isps, IEEE/ACM Transactions On Networking 14 (6) (2006) 1233-1245.

[12] C. Perera, A. Zaslavsky, P. Christen, D. Georgakopoulos, Sensing as a service model for smart cities supported by internet of things, Transactions on Emerging Telecommunications Technologies 25 (1) (2014) 81-93.

[13] U. Aguilera, O. Peña, O. Belmonte, D. L. de Ipiña, Citizen-centric data services for smarter cities, Future Generation Computer Systems 76 (2017) $234-247$.

[14] D. Niyato, X. Lu, P. Wang, D. I. Kim, et al., Economics of Internet of Things: An information market approach, IEEE Wireless Communications (2016) 136-145.

[15] E. Xinhua, J. Han, Y. Wang, L. Liu, Big data-as-a-service: Definition and architecture, in: Communication Technology (ICCT), 2013 15th IEEE International Conference on, IEEE, 2013, pp. 738-742.

[16] D. Niyato, D. T. Hoang, N. C. Luong, P. Wang, D. I. Kim, Z. Han, Smart data pricing models for the internet of things: a bundling strategy approach, IEEE Network 30 (2) (2016) 18-25.

[17] Y. Jiao, P. Wang, D. Niyato, M. A. Alsheikh, S. Feng, Profit maximization auction and data management in big data markets, in: Wireless Communications and Networking Conference (WCNC), 2017 IEEE, IEEE, 2017, pp. 1-6.

[18] D. Ardagna, B. Panicucci, M. Passacantando, Generalized Nash equilibria for the service provisioning problem in cloud systems, Services Computing, IEEE Transactions on 6 (4) (2013) 429-442.

[19] C. Liu, K. Li, C. Xu, K. Li, Strategy configurations of multiple users competition for cloud service reservation, IEEE Transactions on Parallel and Distributed Systems 27 (2) (2016) 508-520.

[20] C. Liu, K. Li, K. Li, R. Buyya, A new cloud service mechanism for profit optimizations of a cloud provider and its users, IEEE Transactions on Cloud Computing doi:10.1109/TCC.2017.2701793.

[21] K. Li, C. Liu, K. Li, A. Y. Zomaya, A framework of price bidding configurations for resource usage in cloud computing, IEEE Transactions on Parallel and Distributed Systems 27 (8) (2016) 2168-2181.

[22] C. Liu, K. Li, K. Li, A game approach to multi-servers load balancing with load-dependent server availability consideration, IEEE Transactions on Cloud Computing doi:10.1109/TCC.2018.2790404.

[23] L. Guijarro, V. Pla, J. R. Vidal, M. Naldi, Game theoretical analysis of service provision for the internet of things based on sensor virtualization, IEEE Journal on Selected Areas in Communications 35 (3) (2017) 691 - 706.

[24] M. E. Ben-Akiva, S. R. Lerman, Discrete choice analysis: theory and application to travel demand, Vol. 9, MIT press, 1985.

[25] P. Maillé, B. Tuffin, Telecommunication network economics: from theory to applications, Cambridge University Press, 2014.

[26] P. Reichl, B. Tuffin, R. Schatz, Logarithmic laws in service quality perception: where microeconomics meets psychophysics and quality of experience, Telecommunication Systems (2011) 1-14.

[27] K. E. Train, Discrete choice methods with simulation, Cambridge University Press, 2009.

[28] S. A. Rhoades, The Herfindahl-Hirschman index, Federal Reserve Bulletin (1993) 188-189.

[29] M. Naldi, Concentration indices and Zipf's law, Economics Letters 78 (3) (2003) $329-334$.

[30] L. Guijarro, M. Naldi, V. Pla, J. R. Vidal, Economic analysis of a centralized brokering platform for wireless sensor data, in: 14th Annual IEEE Consumer Communications and Networking Conference (CCNC), Las Vegas, 2017. 